\title{
Pacific
}

Journal of

Mathematics

\section{ESSENTIAL TORI OBTAINED BY SURGERY ON A KNOT}

MARIO EUdAVE-MUÑOZ 


\section{ESSENTIAL TORI OBTAINED BY SURGERY ON A KNOT}

\section{MARIO EUdAVE-MUÑOZ}

It is proved that if Dehn surgery on a strongly invertible knot, which is not a satellite knot yields a manifold containing an incompressible torus, then the slope of the surgery consists of a certain number of meridians and at most two longitudes. Furthermore, if the slope has two longitudes, then there is an incompressible torus which meets the surgered solid torus twice.

Introduction. Let $k$ be a knot in $S^{3}$, and consider the following construction: Take a solid torus neighborhood $\eta(k)$ of $k$, remove it, and glue it back differently. Let $M_{k}=S^{3}-i n t \eta(k)$. The different regluings are parameterized by the isotopy class $r$, slope, of the simple closed curve on the torus $\partial M_{k}$ that bounds a meridional disk in the reglued solid torus. Denote the resulting closed 3-manifold by $M_{k}(r)$, we say that it is obtained by $r$-Dehn surgery on $k$. Slopes on $\partial M_{k}$ are parameterized by $Q \bigcup\{1 / 0\}$, using a meriaiian-longitude basis $\{\mu, \lambda\}$ for $H_{1}\left(\partial M_{k}\right)$. Then $r$ corresponds to $p / q$ if and only if $[r]=p \mu+q \lambda$ in $H_{1}\left(\partial M_{k}\right) . \Delta(r, s)$ denotes the minimal geometric intersection number of two slopes $r, s$ on $\partial M_{k}$. If $r, s$ correspond to $p / q$ and $a / b$ respectively, then it can be shown that $\Delta(r, s)=$ $|p b-q a|$. For an excellent exposition of the main problems on Dehn surgery on knots see the survey paper of C. McA. Gordon [7].

We consider the following problem: Suppose $k$ is not a satellite knot, i.e. $M_{k}$ does not contain any incompressible, non-boundary parallel torus. When is possible that $M_{k}(r)$ does contain an incompressible torus? i.e. when an essential torus can be created after surgery?

If $k$ is not a satellite and not a torus knot, then by results of W. Thurston [20], $k$ is hyperbolic and $M_{k}(r)$ is hyperbolic for all but 
finitely many $r$. Also, results of S.A. Bleiler and C.G. Hodgson [1] show that if $k$ is hyperbolic then $M_{k}(r)$ has a Riemannian metric of negative curvature for all but at most 24 values of $r$. These results imply that if $k$ is not a satellite knot then $M_{k}(r)$ may contain incompressible tori at most for 24 values of $r$. A result of C.McA. Gordon [8] says that if both $M_{k}(r)$ and $M_{k}(s)$ contain an incompressible torus then $\Delta(r, s) \leq 8$; this also implies that for all but finitely many $r, M_{k}(r)$ does not contain incompressible tori. But these results do not give information about which values of the slope $r$ are possible, in case $M_{k}(r)$ does contain an incompressible torus. In [7], Gordon conjectured that if $M_{k}(r)$ contains an incompressible torus then $\Delta(r, \mu) \leq 2$, in other words, $r$ is homologous to several meridians and at most two longitudes.

In this paper we prove Gordon's conjecture for the case when $k$ is a strongly invertible knot (Theorem 6.3); we show also that if $M_{k}(r)$ contains incompressible tori and $\Delta(r, \mu)=2$, then there is an incompressible torus $T$ in $M_{k}(r)$ which intersects the reglued solid torus in two meridional disks, or in other words, there is a properly embedded, incompressible, punctured torus in $M_{k}$ whose boundary consist of two curves on $\partial M_{k}$ of slope $r$. This last statement can be seen as a kind of generalization of the cabling conjecture, which says that only certain surgery on cable knots yields reducible manifolds, or more explicitly, if $M_{k}(r)$ is reducible then there is a properly embedded, essential annulus on $M_{k}$ whose boundary has slope $r$ on $\partial M_{k}$. There are examples of knots $k$, where $k$ is not satellite, $M_{k}(r)$ contains an incompressible torus for some $r$ so that $\Delta(r, \mu)=2$ and there is an incompressible torus hitting the surgered solid torus twice. The simplest example I know is $37 / 2$-surgery on the $(-2,3,7)$ pretzel knot (see [10]). We have an infinite family of such examples; in those knots there is a non-integral surgery producing a manifold containing an incompressible torus, which hits the surgered solid torus twice, and which divides the manifold into two Seifert fiber spaces with base a disk and 2 exceptional fibers. Those knots also admit two integral surgeries which yield Seifert fiber spaces with base a sphere and at most 3 exceptional fibers. Then for those knots there are 3 different surgeries producing non-hyperbolic manifolds. It is also satisfied that any two of those exceptional surgeries are at distance 1 . Those examples will be explained in a forthcoming 
paper.

C. McA. Gordon and J. Luecke have announced a proof of Gordon's conjecture for all knots. It is still unknown in the general case, if when $\Delta(r, \mu)=2$ there is an incompressible torus hitting the surgered solid torus twice. In the case when $\Delta(r, \mu)=1$ not too much is known; it is not known for example if there is an upper bound for the number of times an incompressible torus may hit the surgered solid torus.

The proof of Theorem 6.3 is somehow inspired by the solution of the cabling conjecture for strongly invertible knots [6]. First, if for a strongly invertible knot $k, M_{k}(r)$ contains an incompressible torus, then by the equivariant torus Theorem of W.H. Holzmann [11], there is an incompressible torus equivariant under the involution of $M_{k}(r)$. By taking quotients, the surgery problem is tranlated into a problem of sums of tangles. In $\S 1$ we state the required results about sums of tangles, which are Theorems 1.3, 1.4 and Corollaries 1.5 and 1.6. In $\S 2,3$ and 4 we prove Theorems 1.3 and 1.4 by using sutured manifold theory and a combinatorial argument. Corollary 1.5 is proved in $\S 5$, and Corollary 1.6 in $\S 1$, for it follows easily from 1.4. Through the paper we assume familiarity with [16] and [6]. In $\S 6$ we apply the Theorems on tangles to get a proof of Theorem 6.3.

\section{Theorems from tangle theory.}

1.1. A tangle $(B, t)$ is a pair that consists of a 3 -ball $B$ and a pair of disjoint arcs and simple closed curves $t$ properly embedded in $B$. Let $B_{1}$ be the unit ball in $R^{3}$, and let $a, b, c, d$, be four points in $\partial B_{1}$ lying in the lines $Y=Z, X=0$ and $Y=-Z, X=0$.

A tangle $\left(B_{1}, t\right)$ is rational if:

(a) It is a trivial tangle, i.e. there is an homeomorphism of pairs from $\left(B_{1}, t\right)$ to the tangle $\left(D^{2} \times I,\{x, y\} \times I\right)$ where $D^{2}$ is the unit ball in $R^{2}$ and $x, y$ are distinct points in the interior of $D^{2}$.

(b) $t \cap \partial B_{1}=\{a, b, c, d\}$.

Two rational tangles $\left(B_{1}, t\right),\left(B_{1}, t^{\prime}\right)$ are equivalent if there is an homeomorphism of pairs $h:\left(B_{1}, t\right) \rightarrow\left(B_{1}, t^{\prime}\right)$ such that $\left.h\right|_{\partial B_{1}}=i d$.

There is a "natural" one to one correspondence between rational tangles and $Q \bigcup\{1 / 0\}$ (see [2], [4], [14]). Denote by $\left(B_{1}, p / q\right)$ the rational tangle determined by $p / q \in Q \bigcup\{1 / 0\}$. As $\left(B_{1}, p / q\right)$ is a trivial tangle, there is a disk $D_{p / q}$ properly embedded in $B_{1}$ which 
separates the strings of $\left(B_{1}, p / q\right)$. Let $J_{p / q}=\partial D_{p / q}$, it is a simple closed curve in $\partial B_{1}-\{a, b, c, d\}$. Define the distance between two rational tangles $\left(B_{1}, p / q\right)$ and $\left(B_{1}, r / s\right)$, denoted by $\Delta(p / q, r / s)$, as half of the minimal number of intersection between the curves $J_{p / q}$ and $J_{r / s}$. It can be shown that $\Delta(p / q, r / s)=|p s-q r|$.

A tangle $(B, t)$ is prime if has the following properties:

(a) It has no local knots, i.e. any $S^{2}$ in $B$ which meets $t$ transversally in two points, bounds in $B$ a ball meeting $t$ in an unknotted spanning arc;

(b) There is no disk properly embedded in $B$ which separates the strings of $(B, t)$;

(c) $B-t$ is irreducible, i.e. any sphere in $B$ disjoint from $t$ bounds a 3-ball disjoint from $t$.

A knot or link $k$ is doubly composite if it can be expressed as the sum of two prime tangles, i.e., there is a sphere $S$ meeting $k$ transversally in four points, such that each of the balls bounded by $S$ determines, with its intersection with $k$, a prime tangle. Such a sphere is called a tangle-decomposing sphere, or simply a decomposing sphere. A knot or link $k$ is doubly prime if it is prime and is not doubly composite. A knot or link $k$ is a satellite knot or link if there is an incompressible torus in $S^{3}-k$ which is not parallel to a component of $\partial \eta(k)$. Such a torus is called a satellite torus.

A Seifert surface for a link $k$ is a compact, orientable surface none of whose components is closed and whose boundary is the link. Define $\chi(k)$ to be the maximal Euler characteristic of all Seifert surfaces for $k$.

1.2. Let $k$ be a link in $S^{3}$. Let $B$ be a 3 -ball in $S^{3}$ which intersects $k$ in two arcs, and such that $(B, B \cap k)$ is a trivial tangle. Suppose also that $\left(B^{\prime}, B^{\prime} \cap k\right)$, where $B^{\prime}=\operatorname{cl}\left(S^{3}-B\right)$, is a prime tangle. Fix an homeomorphism of pairs $h:\left(B_{1}, 1 / 0\right) \rightarrow(B, B \cap k)$. Define a new link $k(B, p / q)$ by changing $(B, B \cap k)$ by $h\left(\left(B_{1}, p / q\right)\right) . k(B, 1 / 0)$ is just $k$. For simplicity denote $h\left(\left(B_{1}, p / q\right)\right)$ by $(B, p / q)$, and $h\left(J_{p / q}\right)$ by $J_{p / q}$.

Let $S$ be a Seifert surface for $k$ with $\chi(S)=\chi(k)$; it is incompressible. $S$ can be isotoped so that it intersects the 3-ball $B$ in a collection of disks; two of them have as boundary one arc of $B \cap k$ plus one arc in $\partial B$; the other disks have as boundary a curve parallel to $J_{1 / 0}$, so $S \cap \partial B$ consist of two arcs and a collection of simple 
closed curves. If $S$ is given an orientation, this induces an orientation on each simple closed curve of $S \cap \partial B$. We say that $B$ intersects $S$ always in the same direction if all the curves $S \cap \partial B$, with the induced orientation, are homologous in $\partial B-h(\{a, b, c, d\})$.

If $k(B, p / q)$ is doubly composite or satellite and $P$ is a decomposing sphere or satellite torus, then $P$ can be isotoped so that it intersects $B$ in a collection of disks, whose boundaries are parallel to the curve $J_{p / q}$ on $\partial B$.

ThEOREM 1.3. Let $k$ be a link and $B$ a 3-ball as before. Suppose that $k(B, p / q)$ is a doubly composite link. Let $P$ be a tangledecomposing sphere for $k(B, p / q)$, isotoped to intersect $(B, p / q)$ in a minimal number of disks. If $\Delta(p / q, 1 / 0)>3$ then one of the following holds

(a) $P$ is disjoint from $B$.

(b) $S^{3}-k$ is irreducible and there is a Seifert surface $S$ for $k$ with $\chi(S)=\chi(k)$, and such that $S$ intersects $\partial B$ only in two arcs, which join the points $h(\{a, b, c, d\})$.

If $\Delta(p / q, 1 / 0)=3$, then either $(a),(b)$, or

(c) $S^{3}-k$ is irreducible and there is a Seifert surface $S$ for $k$ with $\chi(S)=\chi(k)$, and such that $B$ intersects $S$ always in the same direction. Furthermore, $P$ meets $\partial B$ exactly in one curve parallel to $J_{p / q}$.

If $\Delta(p / q, 1 / 0)=2$, then either $(a),(b)$, or

(d) $S^{3}-k$ is irreducible and there is a Seifert surface $S$ for $k$ with $\chi(S)=\chi(k)$, and such that $B$ intersects $S$ always in the same direction. Furthermore, $P$ meets $\partial B$ exactly in two curves parallel to $J_{p / q} ;$ or

(e) $P$ meets $\partial B$ exactly in one curve parallel to $J_{p / q}$.

THEOREM 1.4. Let $k$ be a link and $B$ a 3-ball as before. Suppose that $k(B, p / q)$ is a satellite link, and $T$ is a satellite torus, $T$ not a swallow-follow torus. Suppose $T$ has been isotoped to intersect $(B, p / q)$ in a minimal number of disks. If $\Delta(p / q, 1 / 0)>1$ then one of the following holds

(a) $T$ is disjoint from $B$.

(b) $S^{3}-k$ is irreducible and there is a Seifert surface $S$ for $k$ with $\chi(S)=\chi(k)$, and such that $S$ intersects $\partial B$ only in two arcs.

REMARK. Theorem 1.4 is a generalization of Theorem 3.1 in [18]. 
In our terminology they consider only the case $\Delta=2$.

Corollary 1.5. Let $k, B, k(B, p / q), P$ as in Theorem 1.3. Suppose that $k$ is the trivial knot or a split link. If $\Delta(p / q, 1 / 0) \geq 2$ then one of the following holds

(a) $P$ is disjoint from $B$; or

(b) $\Delta(p / q, 1 / 0)=2$ and $P$ crosses $\partial B$ in one curve parallel to $J_{p / q}$.

Corollary 1.6. Let $k, B, k(B, p / q), T$ as in Theorem 1.4. Suppose that $k$ is the trivial knot or a split link. If $\Delta(p / q, 1 / 0) \geq 2$ then $T$ is disjoint from $B$.

Note that when $\mathrm{k}$ is a split link, cases (b), (c), (d) of Theorem 1.3, and case (b) of Theorem 1.4 cannot happen, i.e. Corollaries 1.5 and 1.6 are obvious when $k$ is a split link. The proof of 1.5 when $k$ is a trivial knot is given in $\S 5$.

Note that Corollaries 1.5 and 1.6 are a kind of generalization of Theorems 2 and 4 in [5], and Theorems 2 and 3 in [6], by replacing the fact of being a composite link by the fact of being a doubly composite or satellite link. Corollaries 1.5 and 1.6 are potentially useful in determining if a given link is doubly prime or non-satellite. We have examples of case (b) of 1.5, which produce via double branched covers, examples for Theorem 6.3 (b). In the case $\Delta=1$ not too much is known; it is not known for example if there is an upper bound for the number of disks of intersection between a tangle decomposing sphere or satellite torus and the ball $B$.

1.7. Proof of Corollary 1.6. Suppose $T$ is a satellite torus in $k(B, p / q)$ isotoped to intersect $(B, p / q)$ a minimum number of times. If (a) of 1.4 happens we are done. If (b) happens then there is a disk $D$ with $\partial D=k$, such that $D$ intersects $\partial B$ only in two arcs. Let $\alpha$ be an arc in $D$ joining two points lying in the distinct arcs of intersection between $D$ and $\partial B$. The two strings of the tangle $\left(B^{\prime}, B^{\prime} \cap k\right)$ are parallel to $\alpha$, so because $\left(B^{\prime}, B^{\prime} \cap k\right)$ is not a trivial tangle, $\alpha$ is a knotted arc in $B^{\prime}$. Let $T_{1}=\partial \eta(B \cup D) . T_{1}$ is an incompressible torus in $S^{3}-k(B, p / q)$, disjoint from $B$. Looking at the curves of intersection between $T$ and $T_{1}$ it is not difficult to conclude that $T$ is disjoint from $B$, except possibly when $\Delta=1$, and $T_{1}$ is the boundary of a neighborhood of a cable knot. 


\section{Preliminary arguments from sutured manifolds.}

2.1. Let $\beta$ be the planar eyeglass 1 -complex consisting of two circles $\beta_{0}$ and $\beta_{1}$ and an arc $\beta_{\alpha}$ joining them. Regard $\beta$ as a complex in $R^{2} \subset R^{3}$, and let $U$ and $W$ be regular neighborhoods of $\beta$ in $R^{2}$ and $R^{3}$ respectively, so that $U$ is a properly embedded planar surface in the genus two handlebody $W . \partial U \subset \partial W$ has three components; two of them, denoted by $c_{0}$ and $c_{1}$, are parallel in $U$ to $\beta_{0}$ and $\beta_{1}$ respectively. Denote the third by $c_{\alpha}$. Denote the cocore of $\beta_{\alpha}$ by $c_{\beta} \subset \partial W$. Let $\lambda_{0}, \lambda_{1}$ be two circles in $\partial W$, parallel to $c_{\beta}$, which bound properly embedded disks in $W$ which separate it in three parts, say a neighborhood of each of $\beta_{0}, \beta_{1}$ and $\beta_{\alpha}$. See Figure 2 in [6].

2.2. Let $k, B$ and $(B, t)$, where $t=B \cap k$, be as in 1.2. Let $W^{\prime}=$ $B$-int $\eta(t)$. Clearly $W^{\prime}$ is homeomorphic to a regular neighborhood of $\beta$; i.e., there is an homeomorphism $f: W \rightarrow W^{\prime}$. Assume that $f\left(c_{0}\right)$ is the cocore of one of the arcs of $t$, and $f\left(c_{1}\right)$ is the cocore of the other arc. Also assume that $f\left(c_{\alpha}\right)=J_{0}$ and $f\left(c_{\beta}\right)=J_{1 / 0}$. For the sake of simplicity we will write $c_{i}$ instead of $f\left(c_{i}\right), i=0,1, \alpha, \beta$ and $\lambda_{i}$ instead of $f\left(\lambda_{i}\right), i=0,1$; that is, consider $\beta$ as embedded in $B$, and $W$ as a neighborhood of $\beta$ in $S^{3}$. The curve $J_{p / q}$ on $\partial W$ intersects the regular neighborhood of $\beta_{0}\left(\beta_{1}\right)$ in a collection of $\Delta=\Delta(p / q, 1 / 0)$ essential arcs, disjoint from $c_{0}\left(c_{1}\right)$, and intersects a neighborhood of $\beta_{\alpha}$ in $2 \Delta$ arcs, each joining $\lambda_{0}$ and $\lambda_{1}$.

2.3. Let $\eta(k)$ be a neighborhood of $k$ disjoint from $W$. Let $M=$ $S^{3}-i n t \eta(k)$. Note that $\beta_{0}$ and $\beta_{1}$ are parallel to meridians of $k$. Note that $\beta \cap \partial M=\emptyset$. Consider $M$ as a sutured manifold, all of whose boundary is in $R_{+}$or $R_{-}$; denote it by $(M, \gamma, \beta)$ (cf. [16]). By hypothesis $S^{3}-(k \cup B)$ is irreducible, for $\left(B^{\prime}, B^{\prime} \cap k\right)$ is a prime tangle; this implies that $(M, \gamma, \beta)$ is $\beta$-irreducible, so $(M, \gamma, \beta)$ is $\beta$-taut. $(M, \gamma)$ may not be $\emptyset$-taut, i.e. it may be reducible or $\partial M$ may be compressible. $\emptyset$ denotes the empty set, to be $\emptyset$-taut means to be taut in the Thurston norm.

The proof of Theorems 1.3 and 1.4 will be as follows: First we take a $\beta$-taut Seifert surface $S$ for $k$, and a decomposing sphere or satellite torus $Q$ for $k(B, p / q)$, which will be considered as a parameterizing surface; then construct a sutured manifold hierarchy, 
starting with $S$ and respecting $Q$. The goal is to prove that the final step in the hierarchy, i.e. $\left(M_{n}, \gamma_{n}\right)$ is $\emptyset$-taut, for in this case $[\mathbf{1 7}, 2.7]$ and $[\mathbf{1 6}, 3.3]$ imply that $(M, \gamma)$ is $\emptyset$-taut unless $k$ is the trivial knot, and $S$ is $\emptyset$-taut, so $\chi(S)=\chi(k), S^{3}-k$ is irreducible and $\beta$ crosses $S$ always in the same direction. In this section we show that $\left(M_{n}, \gamma_{n}\right)$ is $\emptyset$-taut if $Q$ is a torus, and if $Q$ is a decomposing sphere we show the same, except in three cases, one of them being (e) of 1.3. This implies 1.4 and 1.3 (b), (e); the remaining two cases are treated in $\S 3$ and $\S 4$.

2.4. Suppose $k(B, p / q)$ is a doubly composite or a satellite link, and let $P$ be a decomposing sphere or satellite torus, not a swallowfollow torus, for $k(B, p / q)$. Consider $P$ as a properly embedded surface in $S^{3}-\operatorname{int} \eta(k(B, p / q))$; note that $P$ is incompressible and $\partial$-incompressible. $P$ can be isotoped to intersect $(B, p / q)$ in a collection of disks $D_{i}$ properly embedded in $(B, p / q)$, and such that $\partial D_{i}$ is a curve parallel to $J_{p / q}$ in $\partial B$. Assume this number of disks is minimal among all the surfaces isotopic to $P$, and that the intersection is not empty, for otherwise we are done.

Let $Q_{\alpha}=P-i n t B$. Let $(Q, \partial Q) \subset(M-i n t W, \partial M \cup \partial W)$ be a surface consisting of three components, $Q_{0}, Q_{1}$ and $Q_{\alpha}$ with the following properties:

(a) $Q_{0}\left(Q_{1}\right)$ is an annulus for which one boundary component is $c_{0} \subset \partial W\left(c_{1} \subset \partial W\right)$ and the other is a meridian of $\partial \eta(k)$; and either (b) $Q_{\alpha}$ is a connected planar surface, four of its boundary components are meridians in $\partial M$, and the others are parallel to $J_{p / q}$ in $\partial W$; or

(c) $Q_{\alpha}$ is a connected genus one surface, and all of its boundary components are parallel to $J_{p / q}$ in $\partial W$.

According to [16, 7.1], $Q$ is a parameterizing surface for $(M, \gamma, \beta)$. Note that $Q$ is incompressible and, because $P$ is not a swallow-follow torus, $Q$ is also $\partial$-incompressible.

2.5. Let $S$ be a $\beta$-taut Seifert surface for $k$; put $S$ in normal position with respect to $Q([\mathbf{1 6}, 7.2])$. (See $[\mathbf{1 6}, 7.7]$ for the definition of a sutured manifold decomposition respecting a parameterizing surface). It is not difficult to see and is implicit in [16] that the sutured manifold decomposition $(M, \gamma) \stackrel{S}{\longrightarrow}\left(M_{1}, \gamma_{1}\right)$ is $\beta$-taut and 
respects $Q$. Here we use the notion of sutured manifold hierarchy as presented in $[\mathbf{1 7}, 2.1]$. Construct a $\beta$-taut sutured manifold hierarchy

$$
(M, \gamma, \beta) \stackrel{S=S_{1}}{\longrightarrow}\left(M_{1}, \gamma_{1}, \beta_{1}\right) \stackrel{S_{2}}{\longrightarrow} \ldots \stackrel{S_{n}}{\longrightarrow}\left(M_{n}, \gamma_{n}, \beta_{n}\right)
$$

respecting $Q$. (See $[\mathbf{1 7}, 2.5],[\mathbf{1 6}, 4.19 ; 7.8]) . \partial M_{n}$ is a collection of spheres. The surface $S$ meets $\beta_{0}\left(\beta_{1}\right)$ in one point and $Q_{0}\left(Q_{1}\right)$ in one arc. Following $[\mathbf{1 6}, 2.4(\mathrm{c})],[\mathbf{1 7}, 2.1]$, the edges $\beta_{0}, \beta_{1}$ and $\beta_{\alpha}$ can be oriented so that at any point of intersection with an $S_{i}$ (hence with $\left.R\left(\gamma_{i}\right)=R_{+}\left(\gamma_{i}\right) \cup R_{-}\left(\gamma_{i}\right)\right)$ the orientation points in the direction of the normal vector to $S_{i}$. We can suppose $\beta$ has one of the orientations showed in figure 3 in [6] (the choice of one of them depends on the orientation of $\left.S_{1}\right)$. Consider $R_{+}\left(\gamma_{i}\right)\left(R_{-}\left(\gamma_{i}\right)\right)$ as the part of $\partial M_{i}$ in which the orientation points out of (into) $M_{i}$. Denote by $Q_{i}$ and $\beta_{\imath}$ the remnants of $Q$ and $\beta$ in $M_{i}$. Sometimes for simplicity $\beta_{\imath}, R_{+}\left(\gamma_{i}\right), R_{-}\left(\gamma_{\imath}\right), R\left(\gamma_{i}\right)$ will be denoted by $\beta, R_{+}$, $R_{-}, R(\gamma)$ respectively.

2.6. Recall from $[\mathbf{1 6}, 7.4]$ what the index of a parameterizing surface is, $I(Q)=\nu+\mu+K-2 \chi(Q)$, where $\nu$ is the number of sutures and $\mu$ the number of edges that $\partial Q$ crosses. For each arc $\delta$ of $\partial Q \cap \eta(v)$, where $v$ is a vertex of $\beta$, define $\kappa(\delta)$ to be -1 if $\delta$ passes between an edge of $\beta$ pointing into the vertex and one pointing out, and define $\kappa(\delta)=-2$ if $\delta$ passes between edges of $\beta$ both pointing into the vertex or both pointing out (see figure 4 in [6]). Let $K=\sum \kappa$. The curve $J_{p / q} \subset W$ has $\nu=0, \mu=4 \Delta, \kappa=-6 \Delta$, and the curves $c_{0}, c_{1}$ each have $\nu=0, \mu=1, \kappa=-1$. Hence if $Q_{\alpha}$ has $p$ boundary components in $\partial W, \chi\left(Q_{\alpha}\right)=\chi(P)-p$, and so $I\left(Q_{\alpha}\right)=2 p(1-\Delta)-2 \chi(P)$. Also $I\left(Q_{0}\right)=I\left(Q_{1}\right)=0$.

2.7. Suppose that for an arc $\lambda$ of $\beta_{n}$, which contains no vertices, there is a disk $D \subset Q_{n}$, such that $\partial D=\delta_{1} \bigcup \delta_{2}$, where $\delta_{1} \subset \eta(\lambda)$, $\delta_{2} \subset \partial M_{n}$, and $\delta_{2}$ crosses a suture; $\lambda$ is called a cancellable arc, and $D$ is called a cancelling disk. Assume w.l.o.g. that the components of $M_{n}$ which contain a vertex of $\beta$ do not contain cancellable arcs (see $[6,1.6])$.

2.8. Denote by $Q_{i, n}, i=0,1$, the parameterizing surface obtained from $Q_{\imath}$ at the end of the hierarchy. Similarly denote by $\beta_{i, n}, i=$ 
0,1 , the 1-complex obtained from $\beta_{i}$ at the end of the hierarchy. $I\left(Q_{i, n}\right) \leq 0$, by $[\mathbf{1 6}, 7.5 ; 7.6]$.

ClaIM . Each component $q$ of $Q_{i, n}$ for which $\partial Q_{i, n} \bigcap \eta\left(\beta_{i, n}\right) \neq \emptyset$, is a disk for which $I(q)=0$. Indeed $\partial q \cap \beta_{i, n}$ is a single arc and $\partial q \cap \partial M_{n}$ is a single arc crossing a single suture.

The proof is similar to [18, claim 2]. Note that the surface $S_{1}$ meets $\beta_{0}$ and $\beta_{1}$.

This claim implies that there is a component $q$ of $Q_{i, n}, q$ a disk, such that $\partial q$ runs through one of the vertices; all the other components of $Q_{i, n}$ are cancelling disks for an arc of $\beta_{i, n}$.

2.9. A component of $M_{n}$ which does not meet $\beta$ is a 3-ball with a single suture on its boundary. We will disregard these trivial components and suppose with no loss of generality that all the components of $M_{n}$ meet $\beta$. As a consequence of that we conclude that every disk component of $R_{ \pm}$intersects $\beta$. Note also that any component of $\partial M_{n}$ has sutures (see $[6,1.8]$ for details).

$\partial M_{n}$ is a collection of spheres, so $\left(M_{n}, \gamma_{n}\right)$ is $\emptyset$-taut if and only if each sphere has only one suture and is the boundary of a 3-ball. Note that if a component of $M_{n}$ has connected boundary, then this sphere bounds a 3 -ball, for $M_{n}$ is contained in $S^{3}$.

LEMMA 2.10. If none of the surfaces $S_{i}$ intersects $\beta_{\alpha}$ then $\left(M_{n}, \gamma_{n}\right)$ is $\emptyset$-taut.

For the proof see $[6,1.13]$.

2.11. If $\left(M_{n}, \gamma_{n}\right)$ is $\emptyset$-taut then by $[17,2.7]\left(M_{i}, \gamma_{i}\right)$ is $\emptyset$-taut for all $i \geq 1$, and either $(M, \gamma)$ is $\emptyset$-taut and so is irreducible and $\partial$ incompressible, or $M$ is a solid torus (i.e. $k$ is the trivial knot). Now in any case, by $[\mathbf{1 6}, 3.3]$ the surface $S$ is $\emptyset$-taut. It is not difficult to see that this implies that $\chi(S)=\chi(k)$ (for a proof see $[\mathbf{1 9}, 1.2]$ ).

2.12. Suppose that some surface $S_{i}$ intersects $\beta_{\alpha}$. Then there are two components of $\beta_{n}$ which have a vertex. Denote the component which has two ends in $R_{+}\left(R_{-}\right)$and one in $R_{-}\left(R_{+}\right)$by $A_{+}\left(A_{-}\right)$. Denote the ends of $A_{+}$by $a_{+}, a_{-}, b$, and the ends of $A_{-}$by $d_{+}, d_{-}, c$, where $b, c$ are the ends which are part of $\beta_{\alpha} . a_{+}, a_{-}\left(d_{+}, d_{-}\right)$are part of $\beta_{0}\left(\beta_{1}\right) . a_{+}, d_{+}, b$ lie on $R_{+}$, and $a_{-}, d_{-}, c$ lie on $R_{-} .2 .8$ 
shows that $a_{+}$and $a_{-}\left(d_{+}\right.$and $\left.d_{-}\right)$lie in adjacent components of $R_{ \pm}$ and there is a disk $q$ component of $Q_{0, n}\left(Q_{1, n}\right)$ such that $\partial q \cap \partial M_{n}$ is an arc joining $a_{+}$and $a_{-}\left(d_{+}\right.$and $\left.d_{-}\right)$and which crosses a suture. See figure 5 in $[\mathbf{6}]$.

There is a collection of arcs on $A_{+}$going from $a_{+}$to $b$; each such arc $\lambda$ is contained in the boundary of a component of $Q_{n}$, and for this arc $\kappa(\lambda)=-2$. Call the part of $A_{+}$which contains these arcs the negative side of $A_{+}$. Analogously, there is a collection of arcs on $A_{+}$going from $a_{-}$to $b$. For each such arc $\lambda, \kappa(\lambda)=-1$. Call the part of $A_{+}$which contains these arcs the positive side of $A_{+}$. In a similar way we define the negative and positive side of $A_{-}$

Lemma 2.13. Suppose that some surface $S_{i}$ intersects $\beta_{\alpha}$. Then no component of $Q_{n}$ has negative index.

The proof is as in $[6,1.15]$.

2.14. If $\mathrm{P}$ is a torus then $I(Q)=2 p(1-\Delta)<0$ whenever $\Delta>1$. This would contradict lemma 2.13 if some $S_{i}$ meets $\beta_{\alpha}$. So in this case none of the $S_{\imath}$ intersects $\beta_{\alpha}$, and then by 2.10 and 2.11 the surface $S$ is $\emptyset$-taut, $S^{3}-k$ is irreducible and $\chi(S)=\chi(k)$, so we have case (b) of Theorem 1.4. This completes the proof of Theorem 1.4 .

If $P$ is a decomposing sphere then $I(Q)=2 p(1-\Delta)+4$. So $I(Q)<0$ whenever $\Delta>3$. If $\Delta=3$ then $I(Q) \leq 0$, with equality occurring only if $p=1$. If $\Delta=2$ then $I(Q) \leq 2$, and note that $I(Q)=2$ only if $p=1, I(Q)=0$ only if $p=2$, and $I(Q)<0$ if $p>2$. If $I(Q)<0$, then 2.13 implies that none of the $S_{i}$ intersects $\beta_{\alpha}$, and by 2.10 and 2.11 the surface $S$ is $\emptyset$-taut, $\chi(S)=\chi(k)$ and $S^{3}-k$ is irreducible. This implies case (b) of Theorem 1.3. If $\Delta=2$, $I(Q)=2$ and $p=1$ we get case (e) of 1.3 .

From now on we will assume that $P$ is a decomposing sphere, and that $I(Q)=0$, i.e. $\Delta=3$ and $p=1$, or $\Delta=2$ and $p=2$.

REMARK. The above proof for the case $I(Q)<0$ proves essentially the same thing that is proved in [18] and [12]. Instead of using an eyeglass complex they use a complex consisting of the wedge of two circles; it seems that with that complex it is not possible to do the case when $I(Q)=0$. The proof here for the case $I(Q)<0$ is like the one in $[\mathbf{1 6}, \S 8]$. 
3. The combinatorial setting. Our goal in this and next section is to prove that $\left(M_{n}, \gamma_{n}\right)$ is $\emptyset$-taut. We first introduce a combinatorial structure on $\partial M_{n}$, and then prove Lemmas 3.4 and 3.5. These Lemmas are used to prove Lemmas 3.6, 3.7, 3.13, 3.14 and 3.15. Lemmas 3.6 and 3.7 are used in $\S 4$ to show that if $\left(M_{n}, \gamma_{n}\right)$ is not $\emptyset$-taut then there is a finite number of possible configurations of the special vertices (Lemma 4.1). Then it is showed that all these configurations are in contradiction with 3.13-3.15 (Proposition 4.2). This will imply $1.3(\mathrm{c})$ and $(\mathrm{d})$.

3.1. Let $T$ be one of the sphere components of $\partial M_{n}$. The points $\beta \cap T$ and the $\operatorname{arcs} \partial Q_{n} \cap T$ can be regarded as a graph $\Gamma$ in $T$. A vertex of $\Gamma$ is a point of $\beta \cap T$ and an edge is an arc component of $\partial Q_{n} \cap T$, each one of its ends is at a vertex. Denote the components of $\partial Q_{\alpha}-\partial M$ by $a_{1}, a_{2}$ if $\Delta=2$, and by $a_{1}$ if $\Delta=3$. Denote by $\epsilon_{a}=\partial Q_{0, n} \cap T\left(\epsilon_{d}=\partial Q_{1, n} \cap T\right)$ the edge joining $a_{+}$and $a_{-}\left(d_{+}\right.$and $\left.d_{-}\right)$, this edge exists for 2.8 ; it crosses a suture.

$\lambda_{j}$ intersects $a_{i}$ in $2 \Delta$ points, $j=0,1$; label them $i, i^{*}, i, i^{*}, \ldots$ alternately around $\lambda_{j}$, and so that a point $i\left(i^{*}\right)$ in $\lambda_{0}$ is connected, via a subarc of $a_{i}$ contained in $\partial \eta\left(\beta_{\alpha}\right)$, to a point labeled $i\left(i^{*}\right)$ in $\lambda_{1}$. Let $v$ be a vertex in $\Gamma$, then $v$ in $W$ is a circle; label the end of an edge incident to $v$, other than $\epsilon_{a}$ and $\epsilon_{d}$, with $i\left(i^{*}\right)$ if this point is connected to a point in $\lambda_{0}$ labeled with $i\left(i^{*}\right)$, via a subarc of $a_{i}$ whose interior misses $\lambda_{0}, v, a_{ \pm}$and $d_{ \pm}$. See Figure 1 for the case $\Delta=2$, and Figure 2 for the case $\Delta=3$.

Let $\Delta=2$. The curve $a_{1}$ separates $\partial B$ in two parts; one of them

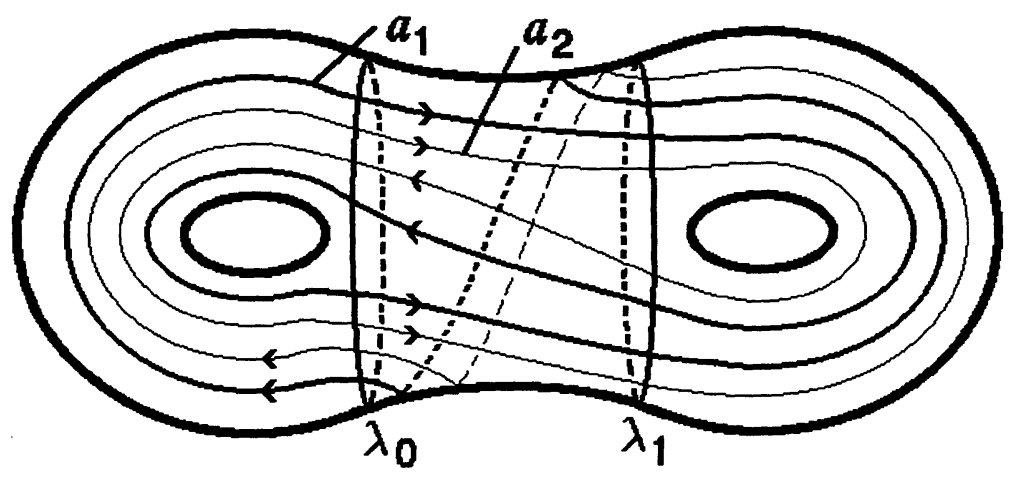

Figure 1. 


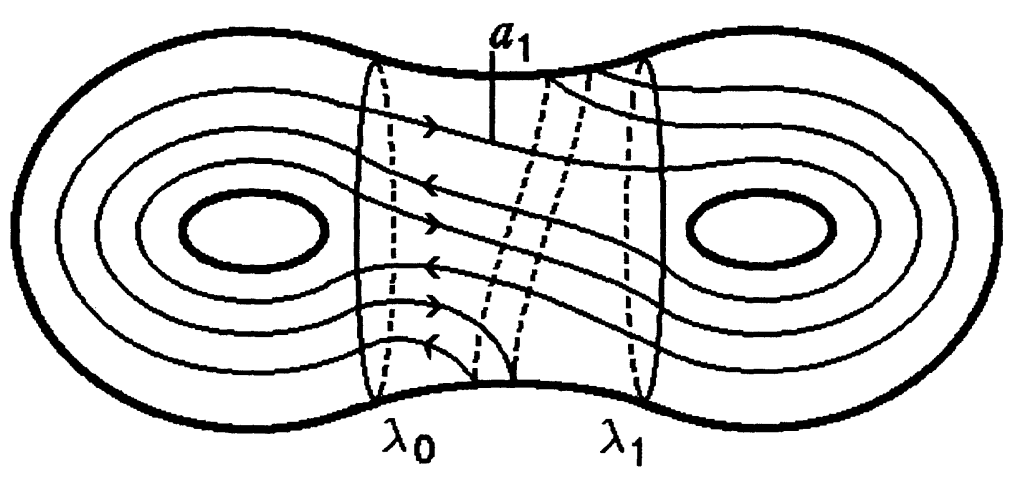

FiguRE 2.

does not contain $a_{2}$, call it $V_{1}$. $V_{1}$ intersects $k$ twice. Choose a rectangle $R_{1} \subset V_{1}$, such that $\partial R_{1}$ consists of four arcs $e_{1}, e_{2}, e_{3}, e_{4}$, where $e_{1}, e_{3}$ are contained in $\partial V_{1}=a_{1}$, and $e_{2}, e_{4}$ are arcs properly embedded in $V_{1}$. Suppose that $R_{1} \cap \eta(k)=\emptyset$, then $R_{1} \subset \partial W$; note that $V_{1}-R_{1}$ has two components, each one of them intersecting $\eta(k)$ once, and assume that for any vertex $v$ in $T, v \neq a_{ \pm}, d_{ \pm}$, we have that $v \cap\left(V_{1}-R_{1}\right)=\emptyset$. It can be assumed that if $v=a_{ \pm}, d_{ \pm}$, then $v \cap \partial V_{1} \subset e_{1} \cup e_{3} \subset \partial R_{1}$. Analogously, there is a disk $V_{2}$ and rectangle $R_{2}$ for $a_{2}$, with $\partial R_{2}=f_{1}, f_{2}, f_{3}, f_{4}$. Label the end of an edge $\epsilon$ in $\Gamma$, labeled previously 1 or $1^{*}$, by $1_{1}$ or $1_{1}^{*}\left(1_{3}\right.$ or $\left.1_{3}^{*}\right)$ if $\epsilon \bigcap a_{1} \subset e_{1}\left(\epsilon \bigcap a_{1} \subset e_{3}\right)$. Analogously change $2,2^{*}$ to $2_{1}, 2_{1}^{*}$ or $2_{3}, 2_{3}^{*}$. It can be assumed w.l.o.g. that the collection of labels around a vertex in $\Gamma$, other than $a_{ \pm}, d_{ \pm}$, looks like $1_{1}, 2_{1}, 2_{3}^{*}, 1_{1}^{*}, 1_{3}, 2_{3}, 2_{1}^{*}, 1_{3}^{*}$. We may assume that $a_{+}$is labeled by $1_{1}, 2_{1}, 2_{3}^{*}, 1_{1}^{*}$, and $a_{-}$is labeled by $1_{3}, 2_{3}, 2_{1}^{*}, 1_{3}^{*}$. See Figure 3 .

Let $\Delta=3$. The curve $a_{1}$ separates $\partial B$ in two parts, $V_{1}$, and $V_{2}$, and there are rectangles $R_{1} \subset V_{1}$, and $R_{2} \subset V_{2}$, as in the previous case. Label the end of an edge $\epsilon$ in $\Gamma$, labeled previously 1 , by $1_{13}$ if $\epsilon \bigcap a_{1} \subset e_{1}$ and $\epsilon \bigcap a_{1} \subset f_{3}$. Analogously change the labels in all the other cases. It can be assumed w.l.o.g. that the collection of labels around a vertex in $\Gamma$, other than $a_{ \pm}, d_{ \pm}$, looks like $1_{13}, 1_{11}^{*}, 1_{33}, 1_{31}^{*}, 1_{11}, 1_{33}^{*}$. To avoid cumbersome notation change these labels for the labels $1,2,3,4,5,6$. We may assume that $a_{+}$is labeled by $1,2,3$, and $a_{-}$is labeled by $4,5,6$. See Figure 4 .

The vertices $a_{ \pm}, d_{ \pm}, b, c$ are named special vertices; any other is called a simple vertex. Call the part of a special vertex which is contained in the negative (positive) side of $A_{+}$or $A_{-}$the negative 


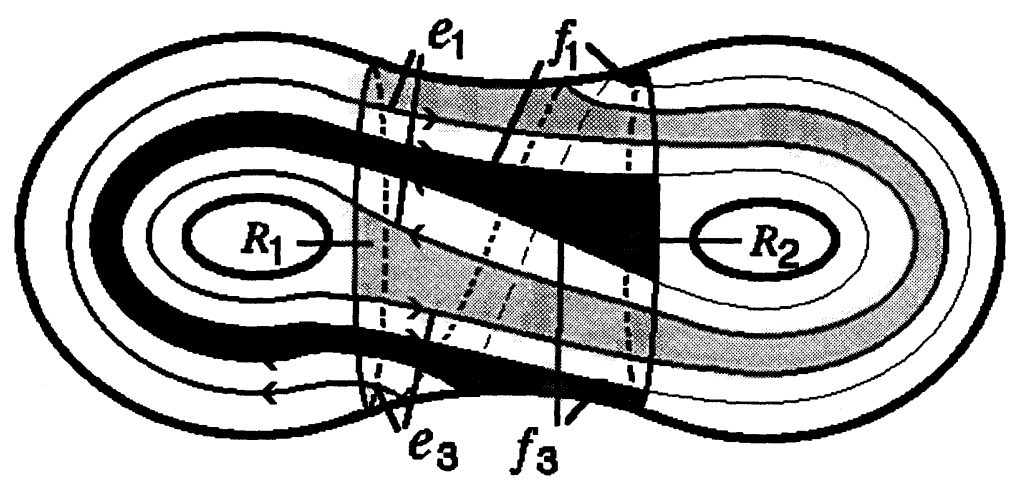

FiguRE 3.

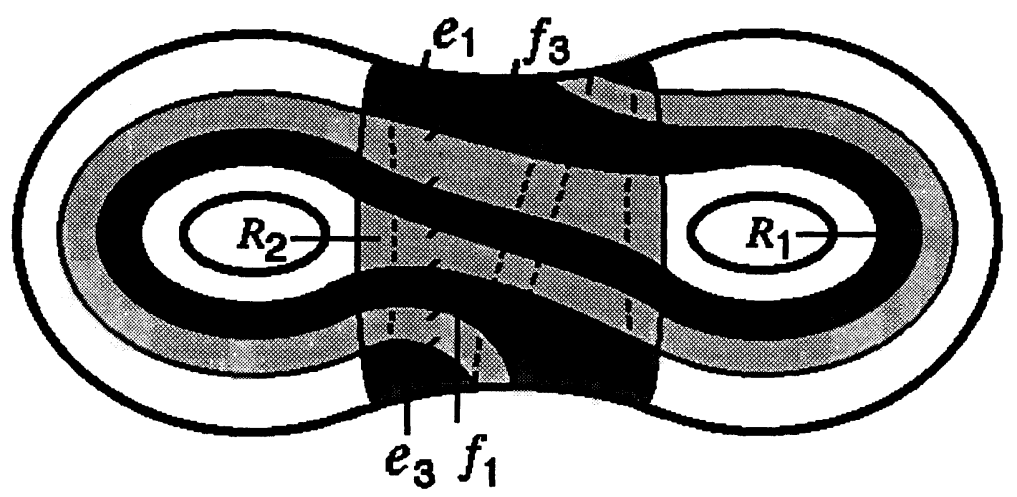

FiguRE 4.

(positive) side of such vertex (see 2.12). $b$ and $c$ have both negative and positive sides, $a_{+}$and $d_{-}$have only a negative side, $a_{-}$and $d_{+}$ have only a positive side.

3.2. Give an orientation to $Q_{\alpha}$; this orientation induces an orientation on the boundary components of $Q_{\alpha}$. Two components of $\partial Q_{\alpha}-\partial M$ are parallel if with the induced orientation they are homologous in $\partial W$; otherwise they are antiparallel. Analogously we define paralellism of vertices in $T$ (see $[6,2.1]$ for the definition). All the vertices of $T$ contained in $R_{+}\left(R_{-}\right)$with the exception of $a_{+}\left(a_{-}\right)$are parallel. A vertex in $R_{+}$is antiparallel to a vertex in $R_{-}$(other than $a_{+}, d_{-}$). The vertex $a_{+},\left(d_{-}\right)$is parallel to vertices in $R_{-}\left(R_{+}\right)$, other than $d_{-}\left(d_{+}\right)$.

We have the following parity rule $[6,2.1]$ : Let $v_{1}, v_{2}$ be vertices of $\Gamma$, and let $i, j \in\{1,2\}$. 
(1) If an edge joins parallel vertices $v_{1}$ and $v_{2}$ with labels $i$ and $j$ $\left(i\right.$ and $j^{*}$, or $i^{*}$ and $j^{*}$ ) respectively, then $a_{i}$ and $a_{j}$ are antiparallel (resp. parallel, resp.antiparallel).

(2) If an edge joins antiparallel vertices $v_{1}$ and $v_{2}$ with labels $i$ and $j\left(i\right.$ and $j^{*}$, or $i^{*}$ and $\left.j^{*}\right)$ respectively, then $a_{i}$ and $a_{j}$ are parallel (resp. antiparallel, resp. parallel).

In the present case if $\Delta=2$ then $\partial Q_{\alpha}-\partial M$ has two components, $a_{1}$ and $a_{2}$, which are antiparallel. If $\Delta=3$, then $\partial Q_{\alpha}-\partial M$ has only one component, i.e. $a_{1}$; in this case the parity rule traduces to: an edge joins parallel vertices $v_{1}$ and $v_{2}$ in $\Gamma$ with labels $i, j \in$ $\{1,2,3,4,5,6\}$, if and only if $i$ and $j$ have different parity.

We make the convention that in all our Figures the vertices parallel to $b$ have labels ordered $1_{1}, 2_{1}, 2_{3}^{*}, 1_{1}^{*}, 1_{3}, 2_{3}, 2_{1}^{*}, 1_{3}^{*}$ or $1,2,3,4,5,6$ in an anticlockwise direction. The vertices antiparallel to $b$ have labels ordered in the opposite direction.

3.3. An edge is level if its ends are equally labeled (ignoring the asterisk and the 1,3 subindex). Define a path and cycle in $\Gamma$ in the usual way.

For $x \in\left\{1_{1}, 2_{1}, 2_{3}^{*}, 1_{1}^{*}, 1_{3}, 2_{3}, 2_{1}^{*}, 1_{3}^{*}\right\}$ or $x \in\{1,2,3,4,5,6\}$, an $x$ path is a path in $\Lambda$ so that the beginning point of each edge is labeled with $x$, and all its vertices are parallel. An $x$-cycle is an $x$-path which is a cycle. An innermost cycle is a cycle which is the boundary of a disk without edges or vertices in its interior. A Scharlemann cycle is an $x$-cycle which is an innermost cycle. Let $\gamma$ be a cycle with edges $\epsilon_{1}, \ldots, \epsilon_{n}$, and vertices $v_{1}, \ldots, v_{n}$, and so that $\epsilon_{i}$ is incident to $v_{i}$ and $v_{i+1}(\bmod \mathrm{n})$, with labels $x_{i}$ and $y_{i}$ respectively; the label sequence of $\gamma$ is the sequence $y_{n}, x_{1}, y_{1}, x_{2}, \ldots, y_{n-1}, x_{n}$.

Let $\Lambda$ be a subgraph of $\Gamma$, and let $x \in\left\{1_{1}, 2_{1}, 2_{3}^{*}, 1_{1}^{*}, 1_{3}, 2_{3}, 2_{1}^{*}, 1_{3}^{*}\right\}$ or $x \in\{1,2,3,4,5,6\}$. We say that $\Lambda$ satisfies $P(x)$ if (see $[3,2.6])$ : For each vertex $v$ in $\Lambda$ there exist an edge of $\Lambda$ incident to $v$ with label $x$ and connecting $v$ to a parallel vertex.

If $\Lambda$ satisfies $P(x)$ for some $x$, then it is possible to construct an $x$-path beginning at any vertex, and this path will close, forming an $x$-cycle in $\Lambda$.

LEMMA 3.4. Let $\Lambda$ be a subgraph of $\Gamma$ which consists of the intersection of $\Gamma$ with a disk, such that all its vertices are parallel, 
and that satisfies $P(x)$ for some $x$. Then $\Gamma$ contains a Scharlemann cycle.

Proof. The proof is a straightforward modification of $[3,2.6 .1$, 2.6.2].

LEMMA 3.5. There is no innermost cycle in $\Gamma$ whose label sequence is one of the following

(a) $1,2,1,2, \ldots, 1,2$, in the case $\Delta=2$, where the asterisk and the subindex have been ignored.

(b) $I_{1}, I_{3}, I_{1}, I_{3}, \ldots, I_{1}, I_{3}$, in the case $\Delta=2$, where $I=1$ or 2 but the same on each label, and the asterisk has been ignored.

(c) $1_{1-}, 1_{3-}, 1_{1-}, 1_{3-}, \ldots, 1_{1-}, 1_{3-}$ or $1_{-1}, 1_{-3}, 1_{-1}, 1_{-3}, \ldots, 1_{-1}, 1_{-3}$, in the case $\Delta=3$, where - stands for 1 or 3 , but not necessarily the same on each label, and the asterisk has been ignored.

In particular there is no Scharlemann cycle in $\Gamma$.

Proof. Suppose $\sigma$ is an innermost cycle, say $\sigma$ consists of vertices $v_{1}, v_{2}, \ldots, v_{n}$, and edges $\epsilon_{1}, \ldots, \epsilon_{n}$, where $\epsilon_{i}$ joins $v_{i}$ and $v_{i+1}(\bmod$ n). There is an arc $\rho_{i}$ in $v_{i}$, joining the labels incident to $\epsilon_{i-1}$ and $\epsilon_{i}$, and which does not contain any label in its interior. Let $D$ be the disk in $T$ bounded by $\sigma$, it follows that $\partial D=\left(\cup \epsilon_{i}\right) \cup\left(\cup \rho_{i}\right)$. If $\sigma$ is a loop then $Q_{\alpha}$ is $\partial$-compressible, which is a contradiction, so suppose $\sigma$ has at least two edges.

If the edges $\epsilon_{i}$ have endpoints labeled 1-2, then all the $\operatorname{arcs} \rho_{i}$ lie over the annulus $E$ cobounded by $a_{1}$ and $a_{2}$, and when traveling along $\sigma$ in a given direction all the $\rho_{i}$ run from $a_{1}$ to $a_{2}$ (or vice versa). The annulus $E$ and the two disks in the interior of $B$ bounded by $a_{1}$ and $a_{2}$ cobound a 3-ball $C \subset B$ whose interior is disjoint from $Q_{\alpha}$. Note that $\partial D \subset Q_{\alpha} \cup E$, and then a regular neighborhood of $Q_{\alpha} \cup C \cup D$ is a punctured lens space, which is impossible.

Suppose the edges $\epsilon_{i}$ are level. First note that the arcs $\rho_{i}$ lie all over $R_{1}$ or all over $R_{2}$. To see that suppose the opposite; then there is an arc $\rho_{i}$ which lies over $R_{1}$ and an $\operatorname{arc} \rho_{j}$ which lies over $R_{2}$, this is possible only if $\Delta=3$. Take two points, one in the interior of each of $\rho_{i}$ and $\rho_{j}$; there is an arc lying over $\partial W$ which joins them and which intersects transversally $Q_{\alpha}$ exactly once, and there is also an arc lying over $D$ joining these points and which is disjoint from $Q_{\alpha}$. So there is a simple closed curve meeting transversally the sphere 
$P$ in one point, which is impossible. Therefore the $\operatorname{arcs} \rho_{i}$ lie all over $R_{1}$ or $R_{2}$, say $R_{1}$. $\rho_{i}$ has its endpoints in $e_{1}$ and $e_{3}$ (or $f_{1}$ and $f_{3}$ ) and when traveling along $\sigma$ in a given direction all the $\rho_{i}$ run from $e_{1}$ to $e_{3}$ (or vice versa). Then $\partial D \subset Q_{\alpha} \cup R_{1}$, and a regular neighborhood of $Q_{\alpha} \cup R_{1} \cup D$ is a punctured lens space, which is not possible.

Finally, note that the label sequence of a Scharlemann cycle is like one of the above sequences.

LEMMA 3.6. Suppose that $D$ is a disk component of $R(\gamma)$ which does not contain $a_{ \pm}$nor $d_{ \pm}$. Let $\Delta=2(\Delta=3)$. Then for each $x \in\left\{1_{1}, 2_{1}, 2_{3}^{*}, 1_{1}^{*}, 1_{3}, 2_{3}, 2_{1}^{*}, 1_{3}^{*}\right\}(x \in\{1,2,3,4,5,6\})$ there is at least one edge with label $x$ at a vertex in $D$ which crosses the suture $\partial D$. So there are at least 8 (6) edges crossing $\partial D$.

Proof. If this does not happen for some $x$ then $\Lambda=D \cap \Gamma$ has $P(x)$, contradicting 3.4 and 3.5 .

LEMMA 3.7. Suppose that $D$ is a disk component of $R(\gamma)$ which contains only one of $a_{ \pm}, d_{ \pm}$. Let $\Delta=2(\Delta=3)$, then there are at least 4 (3) edges which cross $\partial D$, other than $\epsilon_{a}, \epsilon_{d}$.

The proof is as in $[6,2.6]$.

LEMMA 3.8. Let $\Delta=2 . d_{ \pm}$is labeled in one of the following ordered ways:

$$
2_{3}^{*}, 1_{1}^{*}, 1_{3}, 2_{3} ; \quad \text { or } \quad 2_{1}^{*}, 1_{3}^{*}, 1_{1}, 2_{1} .
$$

The positive and negative sides of $c$ are labeled in the same way as $d_{+}$and $d_{-}$respectively.

Proof. The sequence of labels in $d_{+}$has to be a subsequence of $1,2,2^{*}, 1^{*}, 1,2,2^{*}, 1^{*}$. Note that, in the given order of the labels, the first label in $d_{+}$is connected to the last label in $d_{-}$, via a subarc of $a_{1}$ or $a_{2}$ contained in $\partial \eta\left(\beta_{1}\right)$. So one of these labels is 1 and the other $1^{*}$ (or $2^{*}$ and 2 ), see Figure 1. This implies the sequence of labels in $d_{+}$is like $1,2,2^{*}, 1^{*}$ or $2^{*}, 1^{*}, 1,2$. The label $1_{1}$ at $a_{+}$is joined to the label $1_{3}^{*}$ at $a_{-}$, via a subarc of $a_{1}$ contained in $\partial \eta\left(\beta_{0}\right)$; also, these 
two labels are joined, via subarcs of $a_{1}$ contained in $\partial \eta\left(\beta_{\alpha}\right)$ to labels $1_{1}$ and $1_{3}^{*}$ at $d_{ \pm}$. If $d_{+}$has labels $1,2,2^{*}, 1^{*}$, then there is a label $1_{1}$ at $d_{+}$and a label $1_{3}^{*}$ at $d_{-}$(or vice versa), so there is a subarc of $a_{1}$ contained in $\partial \eta\left(\beta_{1}\right)$ which joins these two points. This implies that $a_{1}$ meets $\lambda_{0} \cup \lambda_{1}$ in four points, a contradiction.

LEMMA 3.9. Let $\Delta=3$. $a_{1}$ meets the six points of intersection between $a_{1}$ and $\lambda_{0}$ in one of the following ways, cyclically, 1, 2, 5, 4, 3, 6 or 1,4,3,2,5,6. (The former is shown in Figure 4.)

Proof. Travel around $a_{1}$ starting at the point labeled 1 in $a_{1} \cap \lambda_{0}$, and in the direction of the orientation of $\beta$. Note that the odd and even numbers in $a_{1} \cap \lambda_{0}$ have opposite orientation. It follows from Figure 2 that traveling in that direction, 6 is followed by 1, 2 by 5 , and 4 by 3 . So the sequence of labels in $a_{1}$ has to be one of those written above.

LEMMA 3.10. If $a_{1}$ meets its points of intersection with $\lambda_{0}$ in the cyclic order $1,2,5,4,3,6$ then $d_{+}\left(d_{-}\right)$is labeled $5,6,1(2,3,4)$ or $2,3,4(5,6,1)$. If $a_{1}$ meets its points of intersection with $\lambda_{0}$ in the cyclic order $1,4,3,2,5,6$, then $d_{+}\left(d_{-}\right)$is labeled $3,4,5$ $(6,1,2)$ or $6,1,2(3,4,5)$.

Proof. Note that if a pair of labels, say 1,2 , are consecutive in $\lambda_{0}$ (and then in $\lambda_{1}$ ) and also in $a_{1}$, then one of these labels lies in $d_{+}$, and the other in $d_{-}$. This implies that the labels of $d_{ \pm}$are as desired. See Figure 2.

3.11. Let $D$ be a disk contained in $T$ so that $\partial D$ cuts transversally two special vertices. Suppose all the vertices in $D$ are parallel, except possibly one of the special vertices meeting $\partial D$. Suppose that $a_{ \pm}, d_{ \pm}$are not in the interior of $D$, and that no edge crosses $\partial D$. Let $\Lambda=\Gamma \cap D$. Call the labels of the two vertices which meet $\partial D$, the labels of $\partial D$. Suppose the labels of $\partial D$ in the case $\Delta=2$ are $x_{1}, x_{2}, x_{3}, x_{4}, k_{4}, k_{3}, k_{2}, k_{1}$, in this cyclic order, as shown in Figure 5, where the labels $x_{i}$ correspond to one of the vertices which meet $\partial D$, and the labels $k_{i}$ to the other one. In the case $\Delta=3$ consider six 


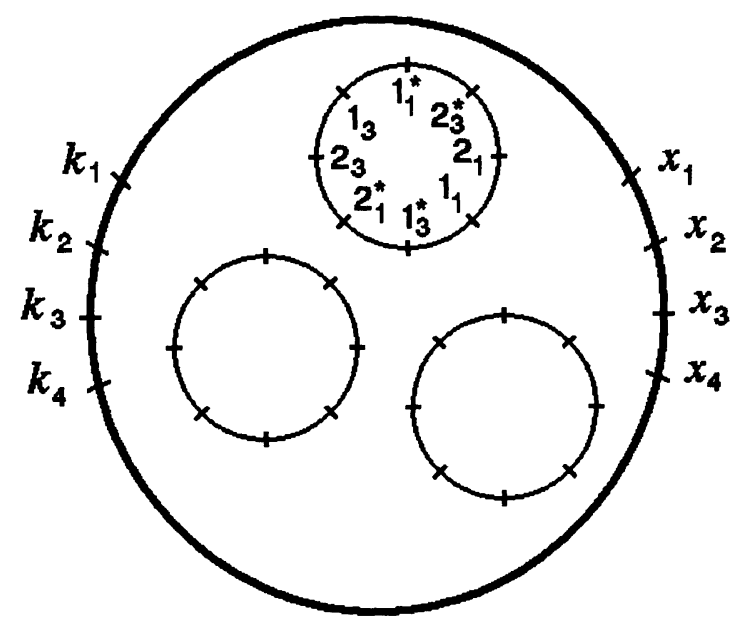

FiguRe 5.

labels in $\partial D$, three for each vertex meeting $\partial D$. Suppose the vertex containing the labels $x_{i}$ 's is parallel to all vertices in the interior of D.

Lemma 3.12. Let $D$ be as in 3.11. Then for each $x_{i}, i=1,2,3,4$, there is a $x_{i}$-path starting at $\partial D$, and finishing at the other side of $\partial D$ with label $k_{i}$.

Proof. For the label $x_{1}$ take a $x_{1}$-path beginning at $\partial D$, this path has to finish at the other side of $\partial D$, otherwise there is a $x_{1}$-cycle, and then by 3.4 a Scharlemann cycle, which contradicts 3.5 , so the path finishes at a label $k_{i_{1}}$ in $\partial D, i_{1} \geq 1$. The complementary part of $\gamma_{1}$ which contains the others labels $x_{i}$ 's has $P\left(x_{2}\right)$. Now take a $x_{2}$-path $\gamma_{2}$ beginning at $\partial D$, it finishes at $\partial D$ with label $k_{i_{2}}$, $i_{2}>i_{1}$. Repeating the argument for $x_{3}$ and $x_{4}$, we conclude that each $x_{i}$-path has to finish in $\partial D$ at the label $k_{i}$.

LEMMA 3.13. Let $\Delta=2$. The following configuration of vertices is not possible: Suppose we have a situation as in 3.11, where $D$ cuts one of $b$ or $a_{ \pm}$, and one of $c$ or $d_{ \pm}$, and the labels of $\partial D$ are in one side the positive or negative side of $b$ or $a_{ \pm}$, and in the other side the positive or negative side of $c$ or $d_{ \pm}$.

Proof. Suppose first that $D$ cuts $b$ and $c$, and assume w.l.o.g. that all the vertices lying in the interior of $D$ are parallel to $b$. 
Therefore $x_{1}, x_{2}, x_{3}, x_{4}$ are $1_{3}, 2_{3}, 2_{1}^{*}, 1_{3}^{*}$ or $1_{1}, 2_{1}, 2_{3}^{*}, 1_{1}^{*}$ respectively, and $k_{1}, k_{2}, k_{3}, k_{4}$ are $2_{3}^{*}, 1_{1}^{*}, 1_{3}, 2_{3}$ or $2_{1}^{*}, 1_{3}^{*}, 1_{1}, 2_{1}$ respectively, for $b$ and $c$ are antiparallel. See Figure 6 . Then by 3.12 for each $i, 1 \leq$ $i \leq 4$, there is a $x_{i}$-path $\gamma_{i}$ starting at $\partial D$ with label $x_{i}$ and finishing at $\partial D$ with label $k_{i}$. Incident to the label $k_{2}\left(=1_{1}^{*}\right.$ or $\left.1_{3}^{*}\right)$ is an edge $\epsilon$ whose other end is at a vertex $v$ with label $2_{3}$ (or $2_{1}$ ). Construct a $1_{3}$-path (or a $1_{1}$-path) $\gamma$ starting at $v$; this path will close forming a cycle or will reach a vertex incident to the path $\gamma_{1}$. So the path $\gamma_{1}-\epsilon-\gamma$ will contain a cycle, and by an argument as in 3.4 there will an innermost cycle $\sigma$ ( $\sigma$ can be chosen to be a Scharlemann cyle if it does not contain $c$, and if it does contain $c$, then can be chosen to be a $x$-cycle, where $x$ is one of the labels $1,1^{*}, 2,2^{*}$, and we have ignored the subindices 1,3 ). If the vertex $c$ is part of the cycle $\sigma$, then all the edges of $\sigma$ have ends labeled 1-2 (i.e. $\sigma$ does not contain level edges, for no edge incident to $c$ is level); if $c$ is not in the cycle $\sigma$, then $\sigma$ is a Scharlemann cycle. Both cases contradict 3.5.

Now suppose $D$ cuts $b$ and $d_{ \pm}$. Then the labels $x_{1}, x_{2}, x_{3}, x_{4}$ correspond to the labels $1_{3}, 2_{3}, 2_{1}^{*}, 1_{3}^{*}$ or $1_{1}, 2_{1}, 2_{3}^{*}, 1_{1}^{*}$, and $k_{1}, k_{2}, k_{3}, k_{4}$ to the labels $2_{3}, 1_{3}, 1_{1}^{*}, 2_{3}^{*}$ or $2_{1}, 1_{1}, 1_{3}^{*}, 2_{1}^{*}$, for $\mathrm{b}$ and $d_{ \pm}$are parallel. Note that in any of the four possible choices of labels there is a label $x$ in common in $b$ and in $d_{ \pm}$, which implies that $\Gamma \cup D$ has $P(x)$, which contradicts 3.4 and 3.5 .

If $D$ cuts $a_{ \pm}$and one of $c$ or $d_{ \pm}$, an argument as in the previous cases yields a contradiction. Note that $a_{ \pm}$is parallel to $c$ and

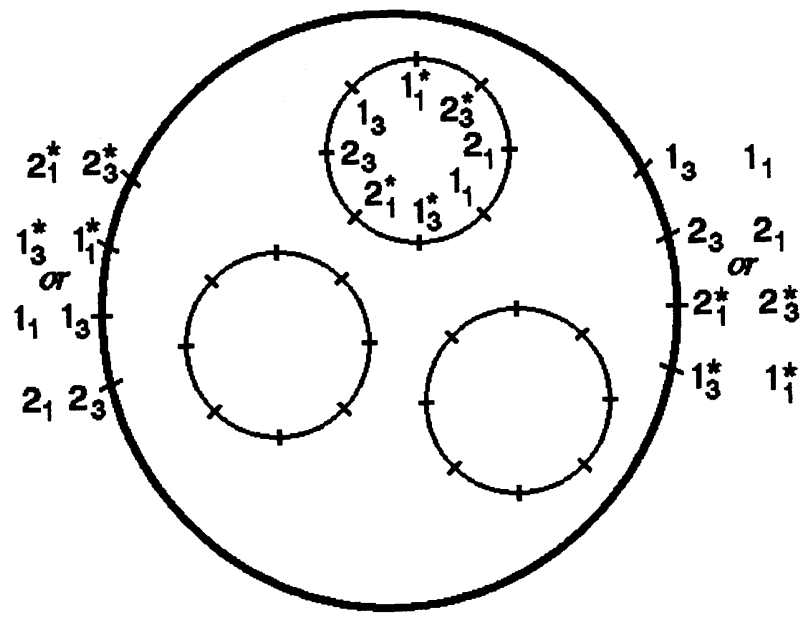

FiguRE 6. 
antiparallel to $d_{ \pm}$.

LEMMA 3.14. Let $\Delta=3$. The following configuration of vertices is not possible: Suppose we have a situation as in 3.11, where $D$ cuts one of $b$ or $a_{ \pm}$and one of $c$ or $d_{ \pm}$, and the labels of $\partial D$ are in one side the positive or negative side of $b$ or $a_{ \pm}$, and in the other side the positive or negative side of $c$ or $d_{ \pm}$.

Proof. Suppose first that $D$ cuts $b$ and $c$, and assume w.l.o.g. all the vertices lying in $D$ are parallel to $b$.

Case 1 . The labels $x_{i}$ 's on $\partial D$ correspond to the negative side of $b$ and the $k_{i}$ 's to the positive or negative side of $c$.

Then $x_{1}, x_{2}, x_{3}$ are $1,2,3$ respectively and $k_{1}, k_{2}, k_{3}$ are $5,6,1$, or $2,3,4$, or $3,4,5$, or $6,1,2$. See Figure 7 . Then by 3.12 , for each $i, i=1,2,3$, there is a $x_{i}$-path $\gamma_{i}$ starting in $\partial D$ at label $x_{i}$ and finishing in $\partial D$ at label $k_{i}$. If $k_{1}, k_{2}, k_{3}$ are $2,3,4$ or $6,1,2$, this will contradict the parity rule, for $b$ and any vertex in the interior of $D$ are antiparallel to $c$. So suppose first $k_{1}, k_{2}, k_{3}$ are $5,6,1$. Let $\epsilon$ be the edge incident to $c$ at label $k_{1}$; the other end of $\epsilon$ is at a vertex $v$ at a label 1. Start a 2-path $\gamma$ at $v$. This path will close forming a cycle, or will reach a vertex incident to the path $\gamma_{2}$. So the path $\gamma_{2}-\epsilon-\gamma$ will contain a cycle, and by an argument as in Lemma 3.4 there will be an innermost cycle $\sigma$. If $c$ is not in $\sigma$, then it will be a Scharlemann cycle, which contradicts Lemma 3.5. If $c$ is part of $\sigma$, so is the arc in $c$ joining the labels $k_{1}$ and $k_{2}$. Remember that $1,2,3$ represent in a short notation the labels $1_{13}, 1_{11}^{*}, 1_{33}$, and $4,5,6$ represent the labels $1_{31}^{*}, 1_{11}, 1_{33}^{*}$. The label sequence of $\sigma$ is like $1,2,1,2, \ldots, 1,2,6,5$, i.e. is like $1_{13}, 1_{11}^{*}, 1_{13}, 1_{11}^{*}, \ldots, 1_{33}^{*}, 1_{11}$. This contradicts 3.5 .

Suppose now $k_{1}, k_{2}, k_{3}$ are $3,4,5$ respectively. Let $\epsilon$ be the edge incident to $c$ at label $k_{3}$; its other end has label 3 at a vertex $v$. Take a 2-path $\gamma$ starting at $v$. Then the path $\gamma_{2}-\epsilon-\gamma$ will form a cycle, and as before there is an innermost cycle $\sigma$. If $c$ is not in $\sigma$, then it is a Scharlemann cycle. If $c$ is in $\sigma$, then $\sigma$ has a label sequence $3,2,3,2, \ldots, 3,2,4,5$, note that this sequence contradicts 3.5 .

Case 2. The labels $x_{i}$ 's on $\partial D$ correspond to the positive side of $b$.

Then $x_{1}, x_{2}, x_{3}$ are 4,5,6 respectively, and as in Case 1, there are four possibilities for the labels $k_{1}, k_{2}, k_{3}$. As in Case 1, the 


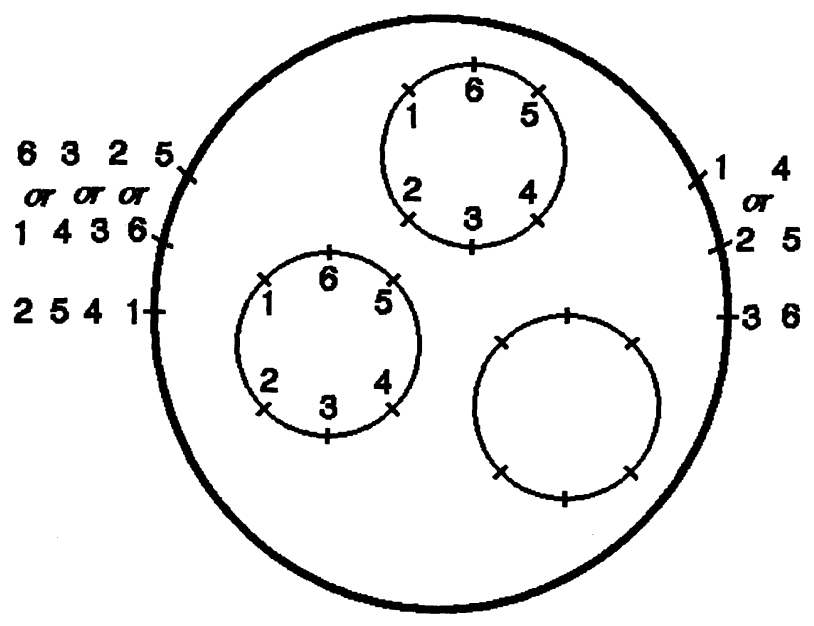

Figure 7.

sequences $5,6,1$ and $3,4,5$ are not possible since they contradict the parity rule. The sequences $2,3,4$ and $6,1,2$ are not possible for they would imply the existence of a lens space summand in $S^{3}$. In the case $2,3,4$ there is an innermost cycle with label sequence $4,5,4,5, \ldots, 3,2$, and in the case $6,1,2$ there is an innermost cycle with label sequence $6,5,6,5, \ldots, 1,2$; both contradict 3.5 .

Suppose now $D$ cuts $b$ and $d_{ \pm}$.

The labels $x_{i}$ 's on $\partial D$ correspond to the negative or positive side of $b$ and the labels $k_{i}$ 's to $d_{+}$or $d_{-}$. There are eight possible choices of labels. Note that in any choice of labels there is a label $x_{i}$ equal to some $k_{j}$, and because $b$ and $d_{ \pm}$are parallel this implies that $\Gamma \cap D$ has $P(x)$ for some label $x$, which contradicts 3.4 and 3.5 .

If $D$ cuts $a_{ \pm}$and one of $c$ or $d_{ \pm}$, an argument as in the previous cases yields a contradiction. Note that $a_{ \pm}$is parallel to $c$ and antiparallel to $d_{ \pm}$.

LEMMA 3.15. The following configuration of vertices is not possible: Suppose we have a situation as in 3.11 , where $D$ cuts $b$ and $a_{ \pm}$, and the labels of $\partial D$ are in one side the positive (negative) side of $b$, and in the other side $a_{+}\left(a_{-}\right)$.

Proof. Let $\Delta=2$. Suppose first that the labels of $\partial D$ correspond to the positive side of $b$ and to $a_{+}$, and assume w.l.o.g. that 
all the vertices lying in the interior of $D$ are parallel to $b$. Therefore $x_{1}, x_{2}, x_{3}, x_{4}$ are $1_{3}, 2_{3}, 2_{1}^{*}, 1_{3}^{*}$ respectively, and $k_{1}, k_{2}, k_{3}, k_{4}$ are $1_{1}, 2_{1}, 2_{3}^{*}, 1_{1}^{*}$ respectively, for $b$ and $a_{+}$are antiparallel. Here we do an argument as in 3.13. By 3.12 for each $i, 1 \leq i \leq 4$, there is a $x_{i}$-path $\gamma_{i}$ starting at $\partial D$ with label $x_{i}$ and finishing at $\partial D$ with label $k_{i}$. Incident to the label $k_{2}=2_{1}$ is an edge $\epsilon$ whose other end is at a vertex $v$ with label $2{ }_{3}$. Construct a $2_{1}^{*}$-path $\gamma$ starting at $v$; this path will close forming a cycle or will reach a vertex incident to the path $\gamma_{3}$. So the path $\gamma_{3}-\epsilon-\gamma$ will contain a cycle, and by an argument as in 3.4 there will an innermost cycle $\sigma$. If the vertex $a_{+}$is part of the cycle $\sigma$, then all the edges of $\sigma$ have ends labeled $2_{3}-2_{1}$ (ignoring the asterisk), if $a_{+}$is not in the cycle $\sigma$, then $\sigma$ is a Scharlemann cycle; both cases contradict 3.5. If the labels of $\partial D$ correspond to the negative side of $b$ and $a_{-}$, a similar argument yields a contradiction.

Let $\Delta=3$. Suppose that the labels of $\partial D$ correspond to the positive side of $b$ and to $a_{+}$, and assume w.l.o.g. that all the vertices lying in the interior of $D$ are parallel to $b$. Therefore $x_{1}, x_{2}, x_{3}$ are 4, 5, 6 respectively, and $k_{1}, k_{2}, k_{3}$, are 1,2,3 respectively, for $b$ and $a_{-}$ are antiparallel. Then as in the previous case there is an edge with ends labeled 4 and 1, which lie on antiparallel vertices, which contradict the parity rule. If the labels of $\partial D$ correspond to the negative side of $b$ and $a_{-}$, a similar argument yields a contradiction.

\section{4. $\left(M_{n}, \gamma_{n}\right)$ is $\emptyset$-taut.}

LEMma 4.1. Suppose that $\left(M_{n}, \gamma_{n}\right)$ is not $\emptyset$-taut. Then $\partial M_{n}$ has at most two boundary components. Suppose first that $\partial M_{n}$ has only one boundary component. This boundary component has two innermost sutures, which determine two disks: one is in $R_{+}$and one in $R_{-}$, denoted by $D_{+}$and $D_{-}$respectively. Denote by $E_{-}, E_{+}$ the annulus incident to $D_{+}$and $D_{-}$respectively. Note that there may be more annuli between $E_{+}$and $E_{-}$. In this case the following are all the possible configurations of the special vertices (see Figure 9 in [6]):

Case 1. $a_{+}$is in $D_{+}, c$ is in $D_{-}, a_{-}$and $d_{-}$are in $E_{-}, b$ and $d_{+}$are in $E_{+}$. 
Case 2. $a_{+}$is in $D_{+}, d_{-}$is in $D_{-}, a_{-}$and $c$ are in $E_{-}, b$ and $d_{+}$are in $E_{+}$.

Case $3 . \quad b$ is in $D_{+}, d_{-}$is in $D_{-}, a_{-}$and $c$ are in $E_{-}, a_{+}$and $d_{+}$are in $E_{+}$.

Case $4 . \quad b$ is in $D_{+}, a_{-}$and $d_{-}$are in $D_{-}, c$ is in $E_{-}, a_{+}$and $d_{+}$are in $E_{+}$.

Case 5. $b$ is in $D_{+}, a_{-}$and $c$ are in $D_{-}, d_{-}$is in $E_{-}, a_{+}$and $d_{+}$are in $E_{+}$.

Case $6 . \quad b$ is in $D_{+}, c$ is in $D_{-}, a_{-}$and $d_{-}$are in $E_{-}, a_{+}$and $d_{+}$are in $E_{+}$.

Case 7. $a_{+}$and $d_{+}$are in $D_{+}, c$ is in $D_{-}, a_{-}$and $d_{-}$are in $E_{-}, \quad b$ is in $E_{+}$.

Case 8. $\quad b$ and $d_{+}$are in $D_{+}, c$ is in $D_{-}, a_{-}$and $d_{-}$are in $E_{-}$, $a_{+}$is in $E_{+}$.

Case $9 . \quad b$ and $d_{+}$are in $D_{+}, a_{-}$and $c$ are in $D_{-}, d_{-}$is in $E_{-}$, $a_{+}$is in $E_{+}$.

Case 10. $a_{+}$and $b$ are in $D_{+}, c$ and $d_{-}$are in $D_{-}, a_{-}$is in $E_{-}, d_{+}$is in $E_{+}$.

Case $11 . \quad d_{+}$is in $D_{+}, a_{-}$is in $D_{-}, c$ and $d_{-}$are in $E_{-}, b$ and $a_{+}$are in $E_{+}$.

Suppose $\partial M_{n}$ has two boundary components, say $T_{1}, T_{2}$; each has only one suture, dividing $T_{1}$ in $D_{1,+}$ and $D_{1,-}$, and $T_{2}$ in $D_{2,+}$ and $D_{2,-}$. The following are all the possible configurations of the special vertices (see Figure 10 in [6]):

Case 12. $b$ and $d_{+}$are in $D_{1,+}, d_{-}$is in $D_{1,-}, a_{+}$is in $D_{2,+}, a_{-}$ and $c$ are in $D_{2,-}$.

Case 13. $b$ is in $D_{1,+}, c$ is in $D_{1,-}, a_{+}$and $d_{+}$are in $D_{2,+}, a_{-}$ and $d_{-}$are in $D_{2,-}$.

Case 14. $a_{+}$and $b$ are in $D_{1,+}, a_{-}$is in $D_{1,-}, d_{+}$is in $D_{2,+}, c$ and $d_{-}$are in $D_{2,-}$.

Proof. Lemmas 3.6, 3.7 and the fact that the negative side of a vertex has 4 or 3 labels imply the following facts, whose proofs are analogous to the corresponding in [6]:

(a) A component $N$ of $\partial M_{n}$ which does not contain a vertex of $\beta$ is a 3 -ball with one suture on its boundary, so is $\emptyset$-taut $[6,3.1]$.

(b) A disk $D$ in $R_{ \pm}$contains special vertices $[6,3.2]$.

(c) A component of $R_{ \pm}$adjacent to a disk also contains a special vertex $[\mathbf{6}, 3.3]$. 
(d) A disk $D$ in $R_{ \pm}$contains at most two special vertices $[6,3.4]$.

(e) A component $T$ of $\partial M_{n}$ has at most three disks components of $R(\gamma)[\mathbf{6}, 3.5]$.

(f) Let $D$ be a disk in $R_{ \pm}$. Suppose that the area adjacent to $D$ is an annulus $E$. Suppose $a_{+}\left(d_{-}\right)$is the only special vertex in $D$. Then $D$ contains simple vertices and $E$ contains either $c$ or $d_{-}$( $b$ or $\left.a_{+}\right)[6,3.6]$.

(g) A component $T$ of $\partial M_{n}$ has at most two innermost sutures $[6,3.8]$.

(h) Let $D$ be a disk in $R_{ \pm}$. Suppose that the area adjacent to $D$ is an annulus $E$. Suppose $a_{-}\left(d_{+}\right)$is in $E$, and $b(c)$ is the only special vertex in $D$. Then either $c$ or $d_{-}\left(b\right.$ or $\left.a_{+}\right)$are in $E[6,3.9]$.

Note however that the proofs of $[6,3.7 ; 3.11]$ do not follow from 3.6, 3.7. Suppose $\partial M_{n}$ has only one component, then claims (a)-(h) almost imply which are all the configurations of the special vertices. Doing an argument similar to [6, 3.12-3.15] we get that Cases 1-11 are all the possible configurations of the special vertices. Cases 10 and 11 do not appear in $[6,3.16]$, but appear here for we are not using $[\mathbf{6}, 3.7 ; 3.11]$.

Suppose $T$ is a component of $\partial M_{n}$. Then doing an argument as in $[6,3.17], T$ cannot meet only $a_{+}$and $a_{-}$, or only $d_{+}$and $d_{-}$. This implies that $\partial M_{n}$ has at most two components. If $\partial M_{n}$ has two components, say $T_{1}$ and $T_{2}$, is not difficult to see that Cases 12, 13, 14 are all the possible configurations of the special vertices. Case 14 appears here and not in $[\mathbf{6}, 3.17]$ because $[\mathbf{6}, 3.7 ; 3.11]$ are not being used.

\section{Proposition 4.2. $\left(M_{n}, \gamma_{n}\right)$ is $\emptyset$-taut.}

Proof. We will show that any of the Cases of 4.1 yields a contradiction.

Case 1. In this Case $a_{+}$is the only special vertex in $D_{+}$. Doing an argument as in $[6,3.6]$, we see that there are simple vertices in $D_{+}$, and no edge incident to $a_{+}$crosses $\partial D_{+}$. Then there are exactly 4 edges (or 3 , depending if $\Delta=2$ or $\Delta=3$ ) crossing $\partial D_{+}$which come from simple vertices. These edges have to meet $d_{-}$. It is not difficult to see that there is a disk $D$ which cuts $a_{ \pm}$and $d_{ \pm}$, as in 3.11 , where the labels of $d_{-}$and $a_{+}$correspond to the labels of $\partial D$. 
Note also that no edge crosses $\partial D$. An application of 3.13 and 3.14 yields a contradiction.

Case 2. As in Case $1, a_{+}$is the only special vertex in $D_{+}$, there are simple vertices in $D_{+}$, and no edge incident to $a_{+}$crosses $\partial D_{+}$. Then there are exactly 4 edges (or 3 , depending if $\Delta=2$ or $\Delta=$ $3)$ crossing $\partial D_{+}$which come from simple vertices. These edges have to meet the negative side of $c$. Let $k_{1}, k_{2}, k_{3}, k_{4}$ be the labels corresponding to the negative side of $c$, ordered according to their cyclical occurrence in $c$. Let $\gamma_{1}\left(\gamma_{4}\right)=$ (edge incident at $c$ at label $\left.k_{1}\left(k_{4}\right)\right) \cap E_{-}$, and let $\alpha$ be the arc in $c$ which goes through the negative side of $c$, and which joins the labels $k_{1}$ and $k_{4}$. There is an $\operatorname{arc} \beta \subset \partial D_{+}$joining the endpoints of $\gamma_{1}$ and $\gamma_{4}$, and so that there is a disk $F \subset E_{-}$, whose boundary is $\partial F=\gamma_{1} \cup \gamma_{4} \cup \alpha \cup \beta$. There are two possibilities:

(1) $a_{-}$lies in $F$. In this Case we have a situation as in 3.11, with $a_{-}$and the positive side of $c$ as the vertices on the boundary of a disk $D$ which contains $F$. An application of 3.13 and 3.14 yields a contradiction.

(2) $a_{-}$does not meet $F$. In this Case there is a disk $D$ as in 3.11, with $a_{+}$and the negative side of $c$ corresponding to the vertices on $\partial D$. An application of 3.13 and 3.14 yields a contradiction.

Case 3. It is similar to Case 1 , with the roles of $d_{-}$and $a_{+}$ interchanged.

Case 4. In this case 8 (or 6) edges cross $\partial D_{+}$, at most 4 (3) of them can reach the negative side of $c$, so there are 4 (3) edges incident to the negative side of $b$ which cross $\partial D_{+}$, and 4 (3) coming from a simple vertex or the positive side of $b$ which have to meet the negative side of $c$. Let $k_{1}, k_{2}, k_{3}, k_{4}$ be the labels corresponding to the negative side of $c$, ordered according to their cyclical ocurrence in $c$. Let $\gamma_{1}\left(\gamma_{4}\right)=$ (edge incident at $c$ at label $\left.k_{1}\left(k_{4}\right)\right) \cap E_{-}$, and let $\alpha$ be the arc in $c$ which goes through the negative side of $c$, and which joins the labels $k_{1}$ and $k_{4}$. There is an arc $\beta \subset \partial D_{+}$joining the endpoints of $\gamma_{1}$ and $\gamma_{4}$, and so that there is a disk $F \subset E_{-}$, whose boundary is $\partial F=\gamma_{1} \cup \gamma_{4} \cup \alpha \cup \beta$. There are two possibilities:

(1) The edges incident to the negative side of $b$ meet $F$. We have a situation as in 3.11, with the negative side of $b$ and the positive side of $c$ as the vertices on the boundary of a disk $D$ which contains $F$. An application of 3.13 and 3.14 yields a contradiction. 
(2) The edges incident to the negative side of $b$ do not meet $F$. Then there is a disk $D$ as in 3.11, with the positive side of $b$ and the negative side of $c$ corresponding to the vertices on $\partial D$. An application of 3.13 and 3.14 yields a contradiction.

Case 5. It is similar to Case 4 , in fact simpler, with $d_{-}$instead of $c$.

Case 7. It is similar to Case 4 , just changing the roles of $b$ and c.

Case 8. It is similar to Case 5 , changing $b$ by $c$, and $d_{-}$by $a_{+}$.

Case 6. In this case doing an argument identical to [6, 4.8] for the same case, we conclude that the 4 (3) edges incident to the positive side of $b$ cross $\partial D_{+}$and meet $a_{-}$, the 4 (3) edges incident to the negative side of $b$ do not cross $\partial D_{+}$, and there are 4 (3) edges incident to simple vertices which cross $\partial D_{+}$and meet $d_{-}$. Then there is a disk which cuts $b$ and $d_{-}$, with the negative side of $b$ and $d_{-}$as the vertices on $\partial D$, and no edge crosses $\partial D$. An application of 3.13 and 3.14 yields a contradiction.

Case 9 . In this case doing an argument as in [6, 4.8], we conclude that there are $4(3)$ edges crossing $\partial D_{+}$, all coming from the negative side of $b$. Then we can find a disk as in 3.11, where $d_{+}$and the positive side of $b$ correspond to the vertices on $\partial D .3 .13$ and 3.14 show that this is not possible.

Case 10. Doing an argument as in [6, 3.11 subclaims 2-4], we get that no edge joins the positive side of $b$ and $a_{-}$, no edge incident to a simple vertex, or to the positive side of $b$, or to $a_{+}$crosses $\partial D_{+}$. Then the 4 (3) edges incident to the negative side of $b$ are the only edges which cross $\partial D_{+}$. So there is a disk $D$ which cuts $b$ and $a_{+}$, as in $3.11, a_{+}$and the positive side of $b$ correspond to the vertices on $\partial D$, and no edge crosses $\partial D$. An application of 3.15 yields a contradiction.

Case 11. Doing an argument as in [6, 3.6 claims 1, 2] we get that there are simple vertices in $D_{-}$, and no edge incident to $a_{-}$ crosses $\partial D_{-}$. So there are 4 (3) edges crossing $\partial D_{-}$, all coming from simple vertices and which have to meet $a_{+}$or the negative side of $b$. An argument as in [6,3.7 claim] shows that none of the edges which cross $\partial D_{-}$meet $a_{+}$, so all have to meet the negative side of $b$. Here as in Cases 2 and 4 there are two possibilities, in one we find a disk as in 3.11 with $a_{-}$and the negative side of $b$ as the vertices 
on $\partial D$, and in the other $a_{+}$and the positive side of $b$ correspond to the vertices on $\partial D$. In both cases an application of 3.15 yields a contradiction.

For Cases 12, 13 and 14 do an argument similar to Cases 9, 4 and 10 respectively.

We have proved that $\left(M_{n}, \gamma_{n}\right)$ is $\emptyset$-taut, then by 2.11 the Seifert surface $S$ is $\emptyset$-taut, so $\chi(S)=\chi(k), S^{3}-k$ is irreducible and $\beta$ always crosses $S$ in the same direction. This implies Cases (c) and (d) of 1.3. This completes the proof of Theorem 1.3.

5. Proof of Corollary 1.5. In this section we prove Corollary 1.5. Let $k$ be the trivial knot, and assume the hypothesis of Theorem 1.3. We want to prove that conclusions (b), (c) and (d) are not possible. Suppose there is a $\beta$-taut disk $S$ with $\partial S=k$. The decomposition $(M, \gamma, \beta) \stackrel{S}{\longrightarrow}\left(M_{1}, \gamma_{1}, \beta_{1}\right)$ is $\beta$-taut, and $M_{1}$ is a 3 ball with only one suture on its boundary, i.e. the sutured manifold hierarchy as constructed in 2.5 consist of only one step. $\partial M_{1}$ is divided into two disks denoted by $D_{+}$and $D_{-}$.

\section{Lemma 5.1. Case (b) of Theorem 1.3 does not happen.}

Proof. If it happens then $\beta_{\alpha}$ does not intersect $S$, so the only vertices in $D_{ \pm}$are $a_{ \pm}, d_{ \pm}$. Then there is a loop at $a_{+}$or $d_{+}$, or there is an edge $\epsilon$ other than $\epsilon_{a}$ incident to $a_{+}$, say, which crosses $\partial D_{+}$. If the former happens then $Q_{\alpha}$ is $\partial$-compressible, a contradiction. If the latter happens then $\epsilon_{a}$ and the edge $\epsilon$ divides $D_{+}$in two regions; one of them, call it $D$, does not contain $d_{+}$. Take an outermost edge incident to $a_{+}$which crosses $\partial D_{+}$; this determines a region, call it $D$ again, which does not contain edges in its interior. It is not difficult to see that, using $D$, the planar surface $P$ can be isotoped to intersect $W$ fewer times.

5.2. Suppose in what follows that either $\Delta=2$ and $p=2$, or $\Delta=3$ and $p=1$. Keep notation as in $\S 3$ and 4 .

Note that there are four points of intersection between $P$ and $k$; this produces four edges, other than $\epsilon_{a}, \epsilon_{d}$, which cross the suture $\partial D_{+}(=k)$. These edges are different, for otherwise there is an 
edge $\epsilon$, say in $D_{+}$, joining two points on $\partial D_{+} \cdot \epsilon$ divides $D_{+}$in two parts; one of them, call it $D$, contains at most one of $a_{+}$or $d_{+}$. If there are vertices in $D$ then $\Gamma \cap D$ has $P(x)$, for some label $x$, which contradict $3.4,3.5$. If there is no vertex in $D$, this gives a $\partial$-compression disk for $Q_{\alpha}$, which is a contradiction.

LEMma 5.3. No edge incident to $a_{ \pm}, d_{ \pm}$crosses the suture $\partial D_{+}$. (other than $\epsilon_{a}, \epsilon_{d}$ ).

Proof. Let $\epsilon$ be an edge incident to $a_{+}$which crosses $\partial D_{+} \cdot \epsilon$ divides $D_{+}$in two regions, one of them, call it $D$, does not contain $d_{+}$. If there are vertices in the interior of $D$ then $\Gamma \cap D$ has $P(x)$ for some label $x$, which contradicts $3.4,3.5$. If there is no vertex inside $D$, do an argument as in 5.1.

Lemma 5.4. Any edge that crosses $\partial D_{+}$is incident to the negative side of $b$ or $c$.

Proof. This is for index restrictions. Let $\epsilon$ be an edge crossing $\partial D_{+} . \epsilon$ is part of the boundary of a disk $q \subset Q_{1}$, with $I(q)=0$, and $\epsilon$ contributes one to the index. If $\epsilon$ does not meet the negative side of a vertex, then it reaches either a simple vertex or the positive side of a special vertex, and the arc of $\partial q$ next to $\epsilon$ contributes one to the index, so either $q$ is a cancelling disk, which contradicts 2.7, or $\epsilon$ is incident to $a_{-}$or $d_{+}$, which contradicts 5.1 .

Lemma 5.5. Let $\epsilon$ be an edge that crosses $\partial D_{+}$. Then the ends of $\epsilon$ are equally labeled.

Proof. This is because $D_{+}$and $D_{-}$form the boundary of a regular neighborhood of the disk $S$ used in the sutured manifold decomposition, and then they look identical.

Lemma 5.6. There are simple vertices in $D_{+}$and $D_{-}$.

Proof. If there is no simple vertex then there are four edges joining $b$ and $c$, and because there is no loop in $\Gamma, 2$ edges (or 1, depending 
if $\Delta=2$ or 3 ) connect each of $a_{+}$and $d_{+}$to $b$, so two edges with consecutive labels connect $a_{+}$and $d_{+}$.

Suppose first that $\Delta=2 . a_{+}$is labeled $1,2,2,1$ and $d_{+}$is labeled $2,1,1,2$. There are two edges connecting $a_{+}$and $d_{+}$with labels 1,2 in $a_{+}$and labels 2,1 in $d_{+}$. These edges form a cycle with label sequence $1,2,1,2$, which contradicts 3.5 .

Suppose that $\Delta=3$. $a_{+}$has labels $3,2,1$, and $d_{+}$has labels $1,6,5$ or $4,3,2$ or $5,4,3$, or $2,1,6$, for $a_{+}$and $d_{+}$are antiparallel. Then there is an edge $\epsilon_{2}$ connecting $a_{+}$and $d_{+}$, with label 2 at $a_{+}$and label $6,3,4$, or 1 at $d_{+}$; note however that if the end of $\epsilon_{2}$ at $d_{+}$ is 1 or 3 , we contradict the parity rule. Let $\epsilon_{1}$ be the other edge connecting $a_{+}$and $d_{+}$. If the ends of $\epsilon_{1}$ and $\epsilon_{2}$ at $a_{+}$are labeled 3 , 2 respectively, and their ends at $d_{+}$are labeled 5,4 or 1,6 , then there is an innermost cycle with label sequence $2,3,5,4$ or $2,3,1$, 6 ; note that both cases are in contradiction with 3.5. If the ends of $\epsilon_{1}$ and $\epsilon_{2}$ at $a_{+}$are labeled 1,2, and their ends at $d_{+}$are labeled 5, 6 , then there is an innermost cycle with label sequence $1,2,6,5$, which contradicts 3.5. Note however that if the ends of $\epsilon_{1}$ and $\epsilon_{2}$ at $a_{+}$are labeled 1, 2, and their ends at $d_{+}$are labeled 3,4 , then the innermost cycle with label sequence $1,2,4,3$ does not contradict 3.5. In this case there is no edge joining $b$ and $c$ with labels 4 , or 5 , for none of these labels is in the negative side of $b$ or $c$, so there are four edges joining $b$ and $c$ with labels $6,1,2,3$. This shows that there are two edges connecting $a_{-}$and $d_{-}$, with labels 6,5 and 2, 1 respectively; these edges form an innermost cycle with label sequence $6,5,1,2$, which does contradict 3.5 .

LEMMA 5.7. No edge joins $b$ and $c$.

Proof. If there is an edge then the argument of 5.5 shows that $b$ and $c$ come from the same point of intersection between $\beta_{\alpha}$ and $S$, i.e. $\beta_{\alpha}$ meets $D$ only once. This implies that there is no simple vertex in $D_{+}$, contradicting 5.6.

LEMMA 5.8. If to a label $x$ in the negative side of $c$ is incident an edge $\epsilon_{1}$ that crosses $\partial D_{-}$, then the edge $\epsilon_{2}$ incident to $d_{-}$at label 
$x$ is level or is incident to the negative side of $c$.

Proof. The edge $\epsilon_{1}$ is part of the boundary of a disk $q \subset Q_{1}$, with $I(q)=0 . \epsilon_{2}$ is part of the same disk $q$. By $5.7, \epsilon_{1}$ is incident to a simple vertex, and then by index restrictions $\epsilon_{2}$ has to meet a simple vertex or the negative side of $c$. In the first case $\epsilon_{2}$ has to be level because $\epsilon_{1}$ is.

Lemma 5.9. Let $\Delta=2$. There is an $x$-cycle in $D_{+}$or $D_{-}$, for some label $x$.

Proof. Because each edge crossing $\partial D_{-}$is incident to the negative side of $b$ or $c$, we can assume w.l.o.g. that there are at least 2 edges incident to the negative side of $c$ which cross $\partial D_{-}$. Suppose the negative (positive) side of $c$ has labels $2_{3}, 1_{3}, 1_{1}^{*}, 2_{3}^{*}\left(2_{1}, 1_{1}, 1_{3}^{*}, 2_{1}^{*}\right)$, as in Figure 8 . Note that no edge in $D_{-}$with an end labeled $1_{3}^{*}$ or $2_{1}^{*}$ can cross $\partial D_{-}$, for none of these labels is in the negative side of $b$ or $c$. If 3 or 4 edges incident to the negative side of $c$ cross $\partial D_{-}$, then 3 or 4 edges incident to $d_{-}$are level or are also incident to the negative side of $c$. Then for at least one of the labels $1_{3}^{*}$ or $2_{1}^{*}$ there is no edge connecting $d_{-}$and a vertex $v$, whose label at $v$ is $1_{3}^{*}$ or $2_{1}^{*}$. This shows that the graph $\Lambda=\left(\Gamma \cap D_{-}\right)-d_{-}$has $P\left(1_{3}^{*}\right)$ or $P\left(2_{1}^{*}\right)$. So suppose exactly two edges incident to the negative side of $c$ cross $\partial D_{-}$.

Suppose the edges incident to the negative side of $c$ which cross $\partial D_{-}$have labels $2_{3}, 1_{3}$ in $c$. The edges incident to $d_{-}$at labels $2_{3}, 1_{3}$ are level or are incident to the negative side of $c$, so none of them has its other end labeled $1_{3}^{*}$ or $2_{1}^{*}$. Let $\epsilon_{1}$ be the edge incident to $d_{-}$at label $1_{3}$; it is also incident to a vertex $v$. Let $\epsilon_{2}$ be the edge incident to $d_{-}$at label $1_{1}^{*}$; if the other endpoint of $\epsilon_{2}$ is not labeled with $1_{3}^{*}$, then $\Lambda$ has $P\left(1_{3}^{*}\right)$, so suppose $\epsilon_{2}$ has that label. Start a $1_{3}^{*}$-path $\gamma$ at the vertex $v$. This path will finish with the edge $\epsilon_{2}$, for otherwise there is a $1_{3}^{*}$-cycle in $D_{-}$. Note that $\epsilon_{1}, \epsilon_{2}$ and $\gamma$ enclose a region which has $P\left(1_{1}\right)$.

There are other possibilities for the labels of the edges incident to the negative side of $c$ which cross the suture $\partial D_{-}$, but a similar argument can be done. If the negative and positive side of $c$ have the other possible labeling, a similar argument is done. 


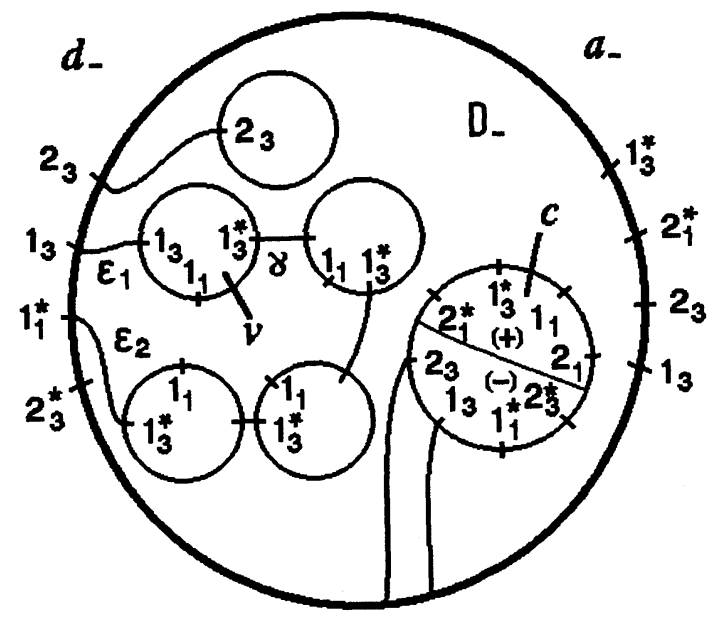

Figure 8.

Lemma 5.10. Let $\Delta=3$. There is an $x$-cycle in $D_{+}$or $D_{-}$, for some label $x$.

Proof. Because each edge crossing $\partial D_{-}$is incident to the negative side of $b$ or $c$, we can assume w.l.o.g. that there are at least 2 edges incident to the negative side of $c$ which cross $\partial D_{-}$. Note that no edge in $D_{-}$with an end labeled $4,5,6$ can cross $\partial D_{-}$, unless it is incident to the negative side of $c$, for none of these labels is in the negative side of $b$. Let $\Lambda$ be as in 5.9.

There are several possibilities for the labeling of the negative side of $c$ and $d_{-}$, namely $1,6,5$ or $2,1,6$ or $4,3,2$, or $5,4,3$. Consider the first two cases; in these cases no edge with a label 4 in $D_{-}$ crosses $\partial D_{-}$, so we look for a 4 -cycle in $\Lambda$. If 3 edges incident to the negative side of $c$ cross $\partial D_{-}$, then all the edges incident to $d_{-}$ are level, by 5.8 , and it follows that $\Lambda$ has $P(4)$. Suppose exactly two edges incident to the negative side of $c$ cross $\partial D_{-}$; note that these edges have consecutive labels in $c$, for otherwise they enclose a region which has $P(4)$ or $P(5)$. If $\Lambda$ does not have $P(4)$, then there is an edge $\epsilon$ joining $d_{-}$and a vertex $v$, whose label at $v$ is 4 , and its label at $d_{-}$is an even number for $d_{-}$is antiparallel to all vertices in $D_{-}$. If $d_{-}$is labelled $1,6,5$, then by the previous observations there is no such edge $\epsilon$. So suppose $d_{-}$is labeled 2, 1,6; the endpoint of $\epsilon$ at $d_{-}$is 2 or 6 , but note that in any case $\Lambda$ has $P(5)$, see Figure 9 . 


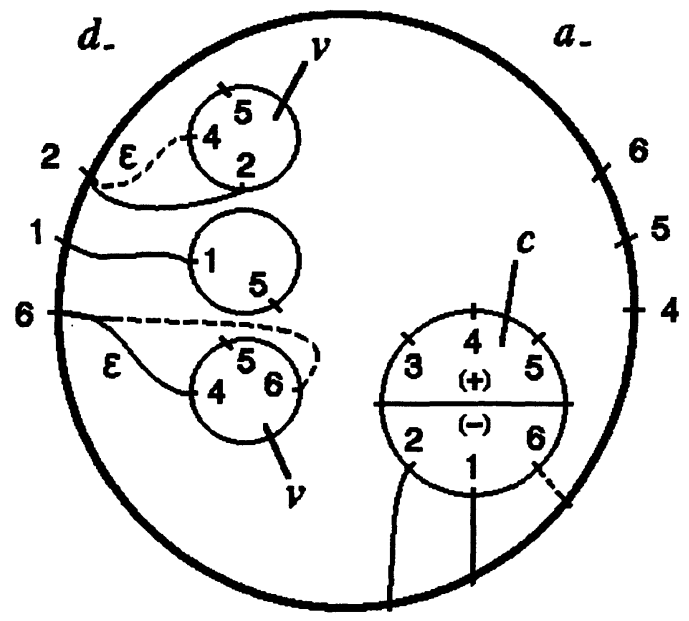

Figure 9.

When the negative side of $c$ has the third or fourth possible labeling, do the same argument for the label 6 .

Clearly 5.9 and 5.10 are in contradiction with 3.4 and 3.5. This completes the proof of Corollary 1.5.

\section{Surgery on strongly invertible knots.}

6.1. Equivariant torus theorem. Let $M$ be an orientable, irreducible 3-manifold with an involution $\tau$. Suppose $M$ contains an incompressible torus. Then one of the following holds:

(1) There is an incompressible torus or Klein bottle $T$ in int $(M)$ transversal to Fix $\tau$ with $T \cap \tau T=\emptyset$ or $\tau T=T$.

(2) $M=V_{-1} \cup V_{1} \cup U_{-1} \cup U_{1}$, where $V_{i}$ and $U_{i}$ are solid tori, $\tau V_{i}=V_{i}$ and $\tau U_{-1}=U_{1}$. There are annuli $A_{i}, i= \pm 1$, with

$$
A_{1} \bigcap A_{-1}=A_{i} \bigcap \tau A_{i}=\partial A_{i}=\partial \tau A_{i}=V_{1} \bigcap V_{-1}=U_{1} \bigcap U_{-1},
$$

and $V_{i} \cap U_{i}=A_{i}, V_{i} \cap U_{-i}=\tau A_{i}, \partial V_{i}=A_{i} \bigcup \tau A_{i}, \partial U_{i}=A_{i} \bigcup \tau A_{-i}$. $A_{1} \cup A_{-1}$ is an incompressible torus transversal to Fix $\tau . \quad \tau \mid V_{i}$ is orientation preserving.

This Theorem follows from $[\mathbf{1 1}, 4.5]$. See Figure 4 in [11] for an illustration of Case 2. Let $N=M / \tau$. If Case (2) of Theorem 6.1 happens then it is not difficult to see that $M$ is a Seifert fiber space over the 2-sphere with 4 exceptional fibers, and that $N$ is a 
lens space, $N \neq S^{1} \times S^{2}, S^{3}$. If Case (1) happens with $T$ being an equivariant Klein bottle, then an Euler characteristic argument shows that $N$ contains either a Klein bottle or a proyective plane. These observations imply the following

COROLlaRY 6.2. Let $M$ be a 3-manifold which is a double cover of $S^{3}$ branched along a link $k$, with deck translation $\tau$. Suppose $M$ contains an incompressible torus. Then there is an incompressible torus $T$ in $M$ which is equivariant, i.e. $\tau T=T$ or $\tau T \cap T=\emptyset$.

THEOREM 6.3. Let $k$ be strongly invertible knot in $S^{3}$, which is not a satellite knot. If $M_{k}(r)$ contains an incompressible torus then

(a) $\Delta(r, \mu) \leq 2$.

(b) If $\Delta(r, \mu)=2$ then there is an incompressible torus in $M_{k}(r)$ which intersects the surgery torus in two disks.

Proof. As $k$ is strongly invertible, there is an involution $\tau$ in $S^{3}$, with $\tau(k)=k$, and $k \cap$ Fix $\tau=\{2$ points $\}$, Fix $\tau$ is an unknotted simple closed curve. A regular neighborhood $\eta(k)$ of $k$ can be chosen so that it is invariant under $\tau$. Then $\tau$ resticts to an involution on $M_{k}=S^{3}-$ int $\eta(k)$. It is not difficult to see that $\tau$ can be extended to an involution $\tau_{r}$ on $M_{k}(r)$ for all $r$.

It follows from [15] that $M_{k}(r) / \tau_{r} \cong S^{3}$, and that the projection $p: M_{k}(r) \rightarrow S^{3}$ is a double cover branched along a link $k_{r}=p($ Fix $\tau)$ of at most two components. The couple $\left(S^{3}, k_{r}\right)$ can be decomposed as the sum of two tangles $\left(B_{2}, t_{2}\right),\left(B_{1}, t_{r}\right)$, where $\left(B_{2}, t_{2}\right)$ is the projection of the exterior of $k$, and $\left(B_{1}, t_{r}\right)$ is the projection of the torus of surgery attached to the exterior of $k .\left(B_{2}, t_{2}\right)$ is a prime tangle, $\left(B_{1}, t_{r}\right)$ is a trivial tangle. $\left(B_{1}, t_{1 / 0}\right)$ can be identified with the rational tangle $\left(B_{1}, 1 / 0\right)$ (cf. 1.1). $\left(S^{3}, k_{1 / 0}\right)$ is the trivial knot; therefore $\left(S^{3}, k_{r}\right)$ can be seen as obtained from $k_{1 / 0}$ by replacing the tangle $\left(B_{1}, 1 / 0\right)$ by the rational tangle $\left(B_{1}, r\right) \cong\left(B_{1}, t_{r}\right)$, as in 1.2.

Assume in what follows that $M_{k}(r)$ is irreducible. There is no loss of generality in doing so, because it is known that only integral surgeries can yield a reducible manifold [9]; further, by the solution of the cabling conjecture for strongly invertible knots [6], if this happened $k$ would be a cable knot, i.e. a satellite knot or a torus knot. 
We are assuming that $M_{k}(r)$ contains an incompressible torus; then by 6.1 there is an incompressible torus $T$, with $\tau T=T$ or $\tau T \cap T=\emptyset$. Suppose first that $\tau T \cap T=\emptyset$. In this case $P=p(T)$ is a torus disjoint from $k_{r} . P$ is incompressible in $S^{3}-k_{r}$, and is not parallel to the boundary of a neighborhood of $k_{r}$, for otherwise $T$ would be compressible. So $P$ is a satellite torus. By Corollary 1.6 , if $\Delta(1 / 0, r)>1$ the torus $P$ can be isotoped to be disjoint from $\left(B_{1}, r\right)$, and then $T$ can be isotoped to be disjoint from $\eta(k)$, i.e. $k$ is a satellite knot, contrary to the hypothesis.

Suppose now that $\tau T=T$. By an Euler characteristic argument $T \cap F i x \tau=\{4$ points $\}$. Then $P=p(T)$ is a sphere intersecting $k_{r}$ in 4 points, i.e. $P$ decomposes $k_{r}$ as the sum of two tangles, which are nontrivial, for otherwise $T$ would be compressible. Both tangles are prime for $M_{k}(r)$ is irreducible and $T$ is incompressible (see [13, Theorem 5]). By Corollary 1.5, if $\Delta(1 / 0, r)>2$ the sphere $P$ can be isotoped to be disjoint from $\left(B_{1}, r\right)$, and then $T$ can be isotoped to be disjoint from $k$, so it is a satellite knot. If $\Delta(1 / 0, r)=$ 2, then $P$ can be isotoped so that $P \cap B_{1}$ consists of an essential disk in $B_{1}-t_{r}$. This implies that $p^{-1}(P \cap B)=T \cap \eta(k)$ consists of two disks; that is, $T$ - int $\eta(k)$ is a genus one surface with two boundary components of slope $r$ on $\partial \eta(k)$, where $\Delta(r, \mu)=2$.

Added in proof: C. McA. Gordon and J. Luecke have recently announced a proof of Theorem 6.3 for all hyperbolic knots.

\section{REFERENCES}

[1] S. A. Bleiler and C. G. Hodgson, Spherical space forms and Dehn surgery, to appear.

[2] J. H. Conway, An enumeration of knots and links, and some of their algebraic properties, Computational problems in abstract algebra, Pergamon Press, Oxford and New York (1969), 324-358.

[3] M. Culler, C. McA. Gordon, J. Luecke and P. Shalen, Dehn Surgery on knots, Ann. of Math., 125 (1987), 237-300.

[4] C. Ernst and D. W. Sumners, A calculus for rational tangles: applications to DNA recombination, Math. Proc. Camb. Phil. Soc., 108 (1990), 489515.

[5] M. Eudave-Muñoz, Primeness and sum of tangles, Trans. Amer. Math. Soc., 306 (1988), 773-790.

[6] M. Eudave-Muñoz, Band sums of links which yield composite links. The cabling conjecture for strongly invertible knots, Trans. Amer. Math. 
Soc., 330 (1992), 463-501.

[7] C. McA. Gordon, Dehn surgery on knots, Proc. of the International Congress of Mathematics, Kyoto, Japan 1990, The Japan Mathematical Society, (1991), 631-642.

[8] C. McA. Gordon, Boundary slopes of punctured tori in 3-manifolds, to appear.

[9] C. McA. Gordon and J. Luecke, Only integral Dehn surgeries can yield reducible manifolds, Math. Proc. Camb. Phil. Soc., 102 (1987), 97-101.

[10] A. Hatcher and U. Oertel, Boundary slopes for Montesinos knots, Topology, 28 (1989), 453-480.

[11] W. H. Holzmann, An equivariant torus Theorem for involutions, Trans. Amer. Math. Soc., 326 (1991), 887-906.

[12] T. Kobayashi, Generalized unknotting operations and tangle decompositions, Proc. Amer. Math. Soc., 105 (1989), 471-478.

[13] W. B. R. Lickorish, Prime knots and tangles, Trans. Amer. Math. Soc., 267 (1981), 321-332.

[14] J .M. Montesinos, Variedades de Seifert que son recubridores cíclicos ramificados de dos hojas, Bol. Soc. Mat. Mexicana (2), 18 (1973), 1-32.

[15] J. M. Montesinos, Surgery on links and double branched covers of $S^{3}$, Annals of Math. Studies no. 84, Princeton Univ. Press, Princeton, N.J., (1975), 227-260.

[16] M. Scharlemann, Sutured manifolds and generalized Thurston norms, J. Differential Geom., 29 (1989), 557-614.

[17] M. Scharlemann, Producing reducible manifolds by surgery on a knot, Topology, 29 (1990), 481-500.

[18] M. Scharlemann and A. Thompson, Unknotting number, genus, and companion tori, Math. Ann., 280 (1988), 191-205.

[19] M. Scharlemann and A. Thompson, Link genus and the Conway moves, Comment. Math. Helvetici, 64 (1989), 527-535.

[20] W. Thurston, Three dimensional manifolds, Kleininan groups and hyperbolic geometry, Bull. Amer. Math. Soc., 6 (1982), 357-381.

Received June 20, 1992 and in revised form December 6, 1992. I am grateful to the Institute for Advanced Study for its hospitality and financial support in the academic year 1990-91, during which this work was begun.

Universidad Nacional Autónoma de MÉXico

Apartado Postal 70-637

MÉXICO, D.F. 04510

MEXICO 
E-mail address: eudave@servidor.unam.mx 



\title{
PACIFIC JOURNAL OF MATHEMATICS
}

Founded by

\author{
E. F. BeCKenBACH (1906-1982) F. Wolf (1904-1989)
}

EDITORS

Sun-Yung A. Chang

(Managing Editor)

University of California

Los Angeles, CA 90024-1555

pacific@math.ucla.edu

F. Michael Christ

University of California

Los Angeles, CA 90024-1555

christ@math.ucla.edu

ThOMAS ENRIGHT

University of California

San Diego, La Jolla, CA 92093

tenright@ucsd.edu
Nicholas ERCOLANI

University of Arizona

Tucson, AZ 85721

ercolani@math.arizona.edu

R. FINN

Stanford University

Stanford, CA 94305

finn@gauss.stanford.edu

VAUGhan F. R. Jones

University of California

Berkeley, CA 94720

vfr@math.berkeley.edu

SteVEn KeRcKHofF

Stanford University

Stanford, CA 94305

spk@gauss.stanford.edu
MARTIN SCHARLEMANN

University of California

Santa Barbara, CA 93106

mgscharl@math.ucsb.edu

\section{GANG TIAN}

Courant Institute

New York University

New York, NY 10012-1110

tiang@taotao.cims.nyu.edu

V. S. VARADARAJAN

University of California

Los Angeles, CA 90024-1555

vsv@math.ucla.edu

\section{SUPPORTING INSTITUTIONS}

UNIVERSITY OF ARIZONA

UNIVERSITY OF BRITISH COLUMBIA

CALIFORNIA INSTITUTE OF TECHNOLOGY

UNIVERSITY OF CALIFORNIA

UNIVERSITY OF MONTANA

UNIVERSITY OF NEVADA, RENO

NEW MEXICO STATE UNIVERSITY

OREGON STATE UNIVERSITY

\author{
UNIVERSITY OF OREGON \\ UNIVERSITY OF SOUTHERN CALIFORNIA \\ STANFORD UNIVERSITY \\ UNIVERSITY OF HAWAII \\ UNIVERSITY OF UTAH \\ WASHINGTON STATE UNIVERSITY \\ UNIVERSITY OF WASHINGTON
}

The Supporting Institutions listed above contribute to the cost of publication of this Journal, but they are not owners or publishers and have no responsibility for its content or policies.

Mathematical papers intended for publication in the Pacific Journal of Mathematics should be in typed form or offset-reproduced (not dittoed), double spaced with large margins. Please do not use built up fractions in the text of the manuscript. However, you may use them in the displayed equations. Underline Greek letters in red, German in green, and script in blue. The first paragraph must be capable of being used separately as a synopsis of the entire paper. In particular it should contain no bibliographic references. Please propose a heading for the odd numbered pages of less than 35 characters. Manuscripts, in triplicate, may be sent to any one of the editors. Please classify according to the 1991 Mathematics Subject Classification scheme which can be found in the December index volumes of Mathematical Reviews. Supply name and address of the author to whom proofs should be sent. All other communications should be addressed to the managing editor, or Julie Honig, University of California, Los Angeles, California 90024-1555.

There are page-charges associated with articles appearing in the Pacific Journal of Mathematics. These charges are expected to be paid by the author's University, Government Agency or Company. If the author or authors do not have access to such Institutional support these charges are waived. Single authors will receive 75 free reprints; joint authors will receive a total of 100 free reprints. Additional copies may be obtained at cost in multiples of 50 .

The Pacific Journal of Mathematics (ISSN 0030-8730) is published monthly except for July and August. Regular subscription rate: $\$ 215.00$ a year (10 issues). Special rate: $\$ 108.00$ a year to individual members of supporting institutions.

Subscriptions, orders for numbers issued in the last three calendar years, and changes of address should be sent to Pacific Journal of Mathematics, P.O. Box 4163, Berkeley, CA 94704-0163, U.S.A. Old back numbers obtainable from Kraus Periodicals Co., Route 100, Millwood, NY 10546.

The Pacific Journal of Mathematics at University of California, c/o Department of Mathematics, 981 Evans Hall, Berkeley, CA 94720 (ISSN 0030-8730) is published monthly except for July and August. Second-class postage paid at Berkeley, CA 94704, and additional mailing offices. POSTMASTER: send address changes to Pacific Journal of Mathematics, P.O. Box 4163, Berkeley, CA 94704-0163.

PUBLISHED BY PACIFIC JOURNAL OF MATHEMATICS at University of California,

Berkeley, CA 94720, A NON-PROFIT CORPORATION

This publication was typeset using $\mathcal{A} \mathcal{M} \mathcal{S}$ - IATEX,

the American Mathematical Society's TEX macro system.

Copyright (C)1994 by Pacific Journal of Mathematics 


\section{PACIFIC JOURNAL OF MATHEMATICS}

Volume $167 \quad$ No. $1 \quad$ January 1995

Local reproducing kernels on wedge-like domains with type 2 edges

Al Boggess and AlexANDER NAGEL

Discriminants of involutions on Henselian division algebras

MaUrice Chacron, H. DHERTE, JEAN-Pierre Tignol,

ADRIAN R. WADSWORTH and V. I. YANCHEVSKIĬ

Essential tori obtained by surgery on a knot

MARIO EUdAVE-MuÑOZ

Non-compact totally peripheral 3-manifolds

LUKE HARRIS and PETER SCOTT

Some representations of TAF algebras

JOHN LINDSAY ORR and JUSTIN PETERS

A non-Haken hyperbolic 3-manifold covered by a surface bundle

ALAN W. REID

The nonhomogeneous minimal surface equation involving a measure 